\title{
Timing the origin of eukaryotic cellular complexity with ancient duplications
}

Julian Vosseberg $^{1 *}$, Jolien J. E. van Hooff ${ }^{1 * \S}$, Marina Marcet-Houben ${ }^{2,3,4}$, Anne van Vlimmeren $^{1 \dagger}$, Leny M. van Wijk ${ }^{1}$, Toni Gabaldón ${ }^{2,3,4,5}$ Berend Snel $^{1}$

${ }^{1}$ Theoretical Biology and Bioinformatics, Department of Biology, Faculty of Science, Utrecht University, Utrecht, The Netherlands

${ }^{2}$ Centre for Genomic Regulation, The Barcelona Institute of Science and Technology, Barcelona, Spain

${ }^{3}$ Life Sciences Department, Barcelona Supercomputing Center, Barcelona, Spain

${ }^{4}$ Mechanisms of Disease, Institute for Research in Biomedicine, Barcelona, Spain

${ }^{5}$ Institució Catalana de Recerca i Estudis Avançats, Barcelona, Spain

*These authors contributed equally to this work

${ }^{\S}$ Current affiliation: Ecologie Systématique Evolution, CNRS, Université Paris-Saclay, AgroParisTech, Orsay, France

${ }^{\dagger}$ Current affiliation: Department of Biological Sciences, Columbia University, New York City, United States of America

Correspondence to: toni.gabaldon@bsc.es (T.G.) or b.snel@uu.nl (B.S.) 
Eukaryogenesis is one of the most enigmatic evolutionary transitions, during which simple prokaryotic cells gave rise to complex eukaryotic cells. While evolutionary intermediates are lacking, gene duplications provide information on the order of events by which eukaryotes originated. Here we use a phylogenomics approach to reconstruct successive steps during eukaryogenesis. We found that gene duplications roughly doubled the proto-eukaryotic gene repertoire, with families inherited from the Asgard archaea-related host being duplicated most. By relatively timing events using phylogenetic distances we inferred that duplications in cytoskeletal and membrane trafficking families were among the earliest events, whereas most other families expanded predominantly after mitochondrial endosymbiosis. Altogether, we infer that the host that engulfed the proto-mitochondrion had some eukaryote-like complexity, which drastically increased upon mitochondrial acquisition. This scenario bridges the signs of complexity observed in Asgard archaeal genomes to the proposed role of mitochondria in triggering eukaryogenesis.

Compared to prokaryotes, eukaryotic cells are tremendously complex. Eukaryotic cells are larger, contain more genetic material, have multiple membrane-bound compartments and operate a dynamic cytoskeleton. Although certain prokaryotes have some eukaryote-like complexity, such as a large size, internal membranes and even phagocytosis-like cell engulfment ${ }^{1,2}$, a fundamental gap remains. The last eukaryotic common ancestor (LECA) already had the intracellular organisation and gene repertoire characteristic of present-day eukaryotes $^{3}$, making the transition from prokaryotes to eukaryotes - eukaryogenesis - one of the main unresolved puzzles in evolutionary biology $y^{1,4}$.

Most eukaryogenesis scenarios posit that a host, related to the recently discovered Asgard archaea ${ }^{5,6}$, took up an Alphaproteobacteria-related endosymbiont ${ }^{7,8}$ that gave rise to the mitochondrion. However, the timing and impact of this endosymbiosis event in the 
evolution of eukaryotic complexity are hotly debated and at the heart of different scenarios on eukaryogenesis 9

Besides the acquisition of genes via the endosymbiont, the proto-eukaryotic genome expanded through gene inventions, duplications and horizontal gene transfers during eukaryogenesis ${ }^{10,11}$. Previous work suggested that gene duplications nearly doubled the ancestral proto-eukaryotic genome ${ }^{11}$. Gene families such as small GTPases, kinesins and vesicle coat proteins greatly expanded, which enabled proto-eukaryotes to employ an elaborate intracellular signalling network, a vesicular trafficking system and a dynamic cytoskeleton $^{12-15}$.

Uncovering the order in which these and other eukaryotic features emerged is complicated due to the absence of intermediate life forms. However, duplications occurred during the transition and are likely to yield valuable insights into the intermediate steps of eukaryogenesis. In this study we attempt to reconstruct the successive stages of eukaryogenesis by systematically analysing large sets of phylogenetic trees inferred from prokaryotic and eukaryotic sequences. We determined the scale of gene inventions and duplications during eukaryogenesis and how different functions and phylogenetic origins had contributed to these eukaryotic innovations. Furthermore, we timed the prokaryotic donations and duplications relative to each other using information from phylogenetic branch lengths.

\section{Results}

\section{Unprecedented resolution of duplications during eukaryogenesis}

To obtain a comprehensive picture of duplications during eukaryogenesis we made use of the Pfam database ${ }^{16}$ (see Methods). We took a phylogenomics approach inspired by the 'ScrollSaw' method ${ }^{14}$, which limits phylogenetic analyses to slowly evolving sequences and 
collapses duplications after LECA and thereby increases the resolution of deep tree nodes. We constructed phylogenetic trees and detected 10,233 nodes in these trees that represent a single Pfam domain in LECA ('LECA families') (Fig. 1a). These 10,233 LECA families do not include genes having only small Pfam domains, which we excluded for computational reasons, or genes without any domains. Therefore, we used a linear regression analysis to obtain an estimated LECA genome containing 12,753 genes (95\% prediction interval: 7,447 21,840) (Extended Data Fig. 1).

Comparing the number of inferred LECA families to extant eukaryotes showed that the genome size of LECA reflected that of a typical present-day eukaryote (Fig. 1a), which is in line with the inferred complexity of LECA, but in contrast with lower estimates obtained previously ${ }^{11,17}$. We used the split between Opimoda and Diphoda as root position of the eukaryotic tree of life ${ }^{18}$. As the exact position of the eukaryotic root is under debate ${ }^{19}$, we tested alternative root positions and obtained very similar numbers of LECA families, except for the root positions at the base of and within the excavates $(15-46 \%$ fewer families compared with an Opimoda-Diphoda root; Extended Data Fig. 2a). In case of a true excavate root, this could reflect fewer genes in LECA. However, given the sampling imbalance between both sides of an excavate root and the reduced nature of sampled excavate genomes, we consider a gene-rich LECA and subsequent gene losses a more likely scenario.

The multiplication factor - the number of LECA families divided by the number of acquired and invented genes or domains - was 1.8, approximating the near doubling reported before $^{11}$. The observed doubling was validated in an additional data set (Supplementary Table 1), despite a recent study that inferred very few duplications during eukaryogenesis (see Supplementary Information $)^{20}$. Although on average genes duplicated once, the distribution of duplications is heavily skewed with many acquisitions from prokaryotes or eukaryotic inventions not having undergone any duplication (Fig. 1b). The enormous expansion of the 
proto-eukaryotic genome was dominated by massive duplications in a small set of families (Supplementary Table 2).

Duplicated and non-duplicated LECA families differed considerably in their functions and cellular localisations. Metabolic LECA families rarely had a duplication history, whereas LECA families involved in information storage and processing, and cellular processes and signalling were more likely to descend from a duplication $\left(\chi^{2}=572, \mathrm{df}=2, \mathrm{P}=7.7 \times 10^{-125}\right.$; Fig. 1c, Supplementary Fig. 1). Notable exceptions to this pattern were families involved in cell wall or membrane biogenesis and translation, which were rarely duplicated. The observed differences in functions were reflected by differences between cellular localisations, with proteins in the endomembrane system and cytoskeleton mostly resulting from a duplication $\left(\chi^{2}=262, \mathrm{df}=4, \mathrm{P}=1.6 \times 10^{-55} ;\right.$ Fig. 1d, Supplementary Fig. 2). Like duplications, inventions primarily occurred to families involved in informational and cellular processes $\left(\chi^{2}=226, \mathrm{df}=\right.$ $2, \mathrm{P}=8.8 \times 10^{-50}$ (function); $\chi^{2}=186, \mathrm{df}=4, \mathrm{P}=4.9 \times 10^{-39}$ (localisation); Extended Data Fig. 3, Supplementary Fig. 3-6). For complex eukaryotes to emerge, most innovations occurred in nuclear processes, the endomembrane system, intracellular transport and signal transduction, especially due to gene duplications.

\section{Relatively large contribution of the host to duplicated LECA families}

For the Pfams that were donated to the eukaryotic stem lineage we identified the prokaryotic sister group, which represents the best candidate for the Pfam's phylogenetic origin (Extended Data Fig. 4a). Most acquisitions had a bacterial sister group (77\%), of which only a small proportion was alphaproteobacterial (7\% of all acquisitions), in agreement with previous analyses $^{10,21,22}$. The acquisitions from archaea (16\%) predominantly had an Asgard archaeal sister (7\% of all acquisitions). Moreover, the most common Asgard archaeal sister group was solely comprised of Heimdallarchaeota (Extended Data Fig. 4b); especially 
Heimdallarchaeote LC3 was frequently the sister group. This is in line with previous analyses providing support for either all Heimdallarchaeota or LC3 being the currently known archaeal lineage most closely related to eukaryotes ${ }^{23,24}$. The species in alphaproteobacterial sister groups, on the other hand, came from different orders (Extended Data Fig. 4c), consistent with the recently proposed deep phylogenetic position of mitochondria ${ }^{8}$. The remaining acquisitions (7\%) had an unclear prokaryotic ancestry (see Supplementary Discussion).

Families with different sister clades varied substantially in the number of gene duplications they experienced during eukaryogenesis $\left(\chi^{2}=50, \mathrm{df}=5, \mathrm{P}=1.2 \times 10^{-9}\right.$ (duplication tendency); $\chi^{2}=190, \mathrm{df}=5, \mathrm{P}=4.3 \times 10^{-39}$ (LECA families from duplication);

Fig. 2). The multiplication factor of 2.2 for families likely inherited from the Asgard archaearelated host was strikingly high compared with the invented families and families acquired from bacteria (between 1.3 and 1.8). Especially duplications related to the ubiquitin system and trafficking machinery contributed to the relatively large number of host-related paralogues (Supplementary Table 2). In contrast, there was a clear deficit of duplications in families with an alphaproteobacterial sister group (multiplication factor of 1.3). Hence, the endosymbiont marginally contributed to the near doubling of the genetic material via duplications during eukaryogenesis, whereas the host contributed relatively the most.

\section{Using branch lengths to time acquisitions and duplications}

The remarkable differences in duplication dynamics between families with different affiliations could tentatively stem from differences in timing of these acquisitions and subsequent duplications. For example, the low number of alphaproteobacterial-associated duplications could be the result of a late mitochondrial acquisition. To research this, branch lengths in phylogenetic trees can be used. They serve as a good proxy for relative time and have previously been used to time the acquisition of genes from the different prokaryotic 
donors ${ }^{10}$. Shorter branch lengths, corrected for differences in evolutionary rates across families, reflect more recent acquisitions. Duplications were not included in the previous analysis, but they can be timed in a similar way with the length of the branch connecting the duplication and LECA nodes (Fig. 3). Although the measure has been criticised for its assumption that evolutionary rates pre- and post-LECA are correlated ${ }^{25,26}$, it yielded correct timings for specific post-LECA events ${ }^{10,27}$. The observed trends can either be created by a common rate change in proteins of the same phylogenetic origin or can be due to different time points of acquisitions. Previous studies ${ }^{10,27}$ showed that the latter explanation is most plausible.

Although the inclusion of duplications in branch length analyses provides potentially valuable information, duplications could have affected the branch lengths by causing a shift in evolutionary rate. The stem lengths of acquisitions that happened simultaneously should approximate the same value, enabling us to assess the effect of duplication on branch lengths. We observed slight but notable increases in stem lengths for duplicated families from alphaproteobacterial origin (Extended Data Fig. 5a) and for more recent duplications in vertebrates (Extended Data Fig. 5f), but not for duplicated families from Asgard archaeal origin (Extended Data Fig. 5b). It is therefore possible that in some families an accelerated rate could result in a slightly too early inferred duplication event according to our branch length analysis. We further checked if there was a rate change after duplication in different functional groups of proteins and looked for an effect of homomer-to-heteromer transitions but we could not detect a clear pattern of rate shifts for different groups of proteins (Extended Data Fig. 5c-e). We validated the use of duplication lengths by examining phylogenetic trees containing more recent duplications in the primate lineage, for which we have multiple intermediate speciation events. The distributions of duplication lengths followed the speciation events (Extended Data Fig. 5g), demonstrating the validity of using duplication 
lengths to obtain an order of events. We also observed a small effect of function but the effect of time was much larger (Extended Data Fig. 5h). Although duplications themselves and function can have an influence, time is the predominant factor explaining the differences in branch lengths. Thus, analysing branch lengths, also in duplicated families, is a valid and effective approach to infer an order of events.

\section{Branch lengths point to a mitochondria-intermediate scenario}

For the timing of acquisitions we obtained similar results as before ${ }^{10}$, with archaeal stems being longer than bacterial stems $\left(\mathrm{P}=4.5 \times 10^{-98}\right.$, two-sided Mann-Whitney $U$-test; Fig. 3). Among the archaeal stem lengths the Asgard archaeal stems were shortest, as were the alphaproteobacterial stems among the bacterial stems, although for the first the difference failed to reach statistical significance $\left(\mathrm{P}=0.88\right.$ and $\mathrm{P}=4.0 \times 10^{-4}$, respectively). This pattern is independent of the normalisation by post-LECA branches, the presence of duplications and functional divergence between the acquisition and LECA (Extended Data Fig. 6). Figure 3 shows that there is a wide distribution of host-related duplication lengths, with a substantial number of duplication lengths both longer and shorter than (alphaproteo)bacterial stem lengths. Bacteria-affiliated, endosymbiont-related and invented families showed the shortest duplication lengths. These duplication lengths were not affected by the position of the

eukaryotic root (Extended Data Fig. 2b). The differences in branch lengths indicate that an increase in genomic complexity via duplications likely had already occurred prior to the mitochondrial acquisition.

To shed light on the evolution of cellular complexity we categorised the duplications according to their functional annotations and cellular localisations. A marked distinction in duplication lengths between different functions can be observed, with duplications in metabolic functions corresponding to shorter branches $\left(\mathrm{P}=8.0 \times 10^{-5}\right.$, Kruskal-Wallis test; 
Fig. 4a, Supplementary Fig. 7). Moreover, a substantial number of duplication lengths in information storage and processes, and cellular processes and signalling functions were longer than the alphaproteobacterial stem length and duplications related to energy production, which mainly involve the mitochondria. These long duplication lengths include multiple duplications assigned to the cytoskeleton and intracellular trafficking. Duplications in signal transduction and transcription families mainly had shorter branch lengths, indicating that these regulatory functions evolved and diversified relatively late. With respect to cellular localisation, nucleolar and cytoskeletal duplication lengths were longest. Most duplications related to the endomembrane system had duplication lengths similar to those of mitochondrial duplications (Fig. 4b, Supplementary Fig. 8). These findings indicate that the increase in cellular complexity before the mitochondrial acquisition mainly comprised the evolution of cytoskeletal, intracellular trafficking and nucleolar components.

\section{Discussion}

This large-scale analysis of duplications during eukaryogenesis provides compelling evidence for a mitochondria-intermediate eukaryogenesis scenario. The results suggest that the Asgard archaea-related host already had some eukaryote-like cellular complexity, such as a dynamic cytoskeleton and membrane trafficking. Upon mitochondrial acquisition there was an even further increase in complexity with the establishment of a complex signalling and transcription regulation network and by shaping the endomembrane system. These postendosymbiosis innovations could have been facilitated by the excess of energy allegedly provided by the mitochondrion ${ }^{28,29}$.

A relatively complex host is in line with the presence of homologues of eukaryotic cytoskeletal and membrane trafficking genes in Asgard archaeal genomes ${ }^{5,6,30}$. Moreover, some of them, including ESCRT-III homologues, small GTPases and (loki)actins, have 
duplicated in these archaea as well, either before eukaryogenesis or more recently ${ }^{5,6,30}$. This indicates that there has already been a tendency for at least the cytoskeleton and membrane remodelling to become more complex in Asgard archaeal lineages. A dynamic cytoskeleton and trafficking system, perhaps enabling primitive phagocytosis ${ }^{31}$, might have been essential for the host to take up the bacterial symbiont. Molecular and cell biology research in these archaea, from which the first results have recently become public ${ }^{32,33}$, is highly promising to yield more insight into the nature of the host lineage. In addition to a reconstruction of the host, further exploration of the numerous acquisitions, inventions and duplications during eukaryogenesis is key to fully unravelling the origin of eukaryotes.

\section{References}

1. Dacks, J. B. et al. The changing view of eukaryogenesis - fossils, cells, lineages and how they all come together. J. Cell Sci. 129, 3695-3703 (2016).

2. Shiratori, T., Suzuki, S., Kakizawa, Y. \& Ishida, K. Phagocytosis-like cell engulfment by a planctomycete bacterium. Nat. Commun. 10, 1-11 (2019).

3. Koumandou, V. L. et al. Molecular paleontology and complexity in the last eukaryotic common ancestor. Crit. Rev. Biochem. Mol. Biol. 48, 373-396 (2013).

4. Szathmáry, E. Toward major evolutionary transitions theory 2.0. Proc. Natl. Acad. Sci. U. S. A. 112, 10104-10111 (2015).

5. Spang, A. et al. Complex archaea that bridge the gap between prokaryotes and eukaryotes. Nature 521, 173-179 (2015).

6. Zaremba-Niedzwiedzka, K. et al. Asgard archaea illuminate the origin of eukaryotic cellular complexity. Nature 541, 353-358 (2017).

7. Roger, A. J., Muñoz-Gómez, S. A. \& Kamikawa, R. The origin and diversification of mitochondria. Curr. Biol. 27, R1177-R1192 (2017). 
8. Martijn, J., Vosseberg, J., Guy, L., Offre, P. \& Ettema, T. J. G. Deep mitochondrial origin outside the sampled alphaproteobacteria. Nature 557, 101-105 (2018).

9. Poole, A. M. \& Gribaldo, S. Eukaryotic origins: how and when was the mitochondrion acquired? Cold Spring Harb. Perspect. Biol. 6, a015990 (2014).

10. Pittis, A. A. \& Gabaldón, T. Late acquisition of mitochondria by a host with chimaeric prokaryotic ancestry. Nature 531, 101-104 (2016).

11. Makarova, K. S., Wolf, Y. I., Mekhedov, S. L., Mirkin, B. G. \& Koonin, E. V. Ancestral paralogs and pseudoparalogs and their role in the emergence of the eukaryotic cell. Nucleic Acids Res. 33, 4626-4638 (2005).

12. Jékely, G. Small GTPases and the evolution of the eukaryotic cell. BioEssays 25, 1129-1138 (2003).

13. Wickstead, B., Gull, K. \& Richards, T. A. Patterns of kinesin evolution reveal a complex ancestral eukaryote with a multifunctional cytoskeleton. BMC Evol. Biol. 10, 110 (2010).

14. Elias, M., Brighouse, A., Gabernet-Castello, C., Field, M. C. \& Dacks, J. B. Sculpting the endomembrane system in deep time: high resolution phylogenetics of Rab GTPases. $J$. Cell Sci. 125, 2500-2508 (2012).

15. Dacks, J. B. \& Field, M. C. Evolutionary origins and specialisation of membrane transport. Curr. Opin. Cell Biol. 53, 70-76 (2018).

16. Finn, R. D. et al. The Pfam protein families database: towards a more sustainable future. Nucleic Acids Res. 44, D279-D285 (2016).

17. Fritz-Laylin, L. K. et al. The genome of Naegleria gruberi illuminates early eukaryotic versatility. Cell 140, 631-642 (2010).

18. Derelle, R. et al. Bacterial proteins pinpoint a single eukaryotic root. Proc. Natl. Acad. Sci. U. S. A. 112, E693-E699 (2015). 
19. Burki, F., Roger, A. J., Brown, M. W. \& Simpson, A. G. B. The new tree of eukaryotes. Trends Ecol. Evol. 35, 43-55 (2020).

20. Tria, F. D. K. et al. Gene duplications trace mitochondria to the onset of eukaryote complexity. Preprint at http://www.biorxiv.org/content/10.1101/781211v1 (2019).

21. Esser, C. et al. A genome phylogeny for mitochondria among $\alpha$-proteobacteria and a predominantly eubacterial ancestry of yeast nuclear genes. Mol. Biol. Evol. 21, 1643-1660 (2004).

22. Pisani, D., Cotton, J. A. \& McInerney, J. O. Supertrees disentangle the chimerical origin of eukaryotic genomes. Mol. Biol. Evol. 24, 1752-1760 (2007).

23. Narrowe, A. B. et al. Complex evolutionary history of translation elongation factor 2 and diphthamide biosynthesis in archaea and parabasalids. Genome Biol. Evol. 10, 2380-2393 (2018).

24. Williams, T. A., Cox, C. J., Foster, P. G., Szöllősi, G. J. \& Embley, T. M. Phylogenomics provides robust support for a two-domains tree of life. Nat. Ecol. Evol. 4, $138-147$ (2020).

25. Martin, W. F. et al. Late mitochondrial origin is an artifact. Genome Biol. Evol. 9, 373-379 (2017).

26. Lane, N. Serial endosymbiosis or singular event at the origin of eukaryotes? J. Theor. Biol. 434, 58-67 (2017).

27. Pittis, A. A. \& Gabaldón, T. On phylogenetic branch lengths distribution and the late acquistion of mitochondria. Preprint at https://www.biorxiv.org/content/10.1101/064873v1 (2016).

28. Lane, N. \& Martin, W. The energetics of genome complexity. Nature 467, 929-934 (2010).

29. Lane, N. Bioenergetic constraints on the evolution of complex life. Cold Spring Harb. 
Perspect. Biol. 6, a015982 (2014).

30. Klinger, C. M., Spang, A., Dacks, J. B. \& Ettema, T. J. G. Tracing the archaeal origins of eukaryotic membrane-trafficking system building blocks. Mol. Biol. Evol. 33, 1528-1541 (2016).

31. Martijn, J. \& Ettema, T. J. G. From archaeon to eukaryote: the evolutionary dark ages of the eukaryotic cell. Biochem. Soc. Trans. 41, 451-457 (2013).

32. Ak1l, C. \& Robinson, R. C. Genomes of Asgard archaea encode profilins that regulate actin. Nature 562, 439-443 (2018).

33. Imachi, H. et al. Isolation of an archaeon at the prokaryote-eukaryote interface. Nature 577, 519-525 (2020).

34. Deutekom, E. S., Vosseberg, J., Dam, T. J. P. van \& Snel, B. Measuring the impact of gene prediction on gene loss estimates in Eukaryotes by quantifying falsely inferred absences. PLOS Comput. Biol. 15, e1007301 (2019).

35. Huerta-Cepas, J. et al. eggNOG 4.5: a hierarchical orthology framework with improved functional annotations for eukaryotic, prokaryotic and viral sequences. Nucleic Acids Res. 44, D286-D293 (2016).

36. Eddy, S. R. Accelerated Profile HMM Searches. PLOS Comput. Biol. 7, e1002195 (2011).

37. Hauser, M., Mayer, C. E. \& Söding, J. kClust: fast and sensitive clustering of large protein sequence databases. BMC Bioinformatics 14, 248 (2013).

38. Wijk, L. M. van \& Snel, B. The first eukaryotic kinome tree illuminates the dynamic history of present-day kinases. Preprint at https://www.biorxiv.org/content/10.1101/2020.01.27.920793v2 (2020).

39. Altschul, S. F., Gish, W., Miller, W., Myers, E. W. \& Lipman, D. J. Basic local alignment search tool. J. Mol. Biol. 215, 403-410 (1990). 
40. Shah, N., Nute, M. G., Warnow, T. \& Pop, M. Misunderstood parameter of NCBI BLAST impacts the correctness of bioinformatics workflows. Bioinformatics 35, 1613-1614 (2019).

41. González-Pech, R. A., Stephens, T. G. \& Chan, C. X. Commonly misunderstood parameters of NCBI BLAST and important considerations for users. Bioinformatics 35, 26972698 (2019).

42. Adl, S. M. et al. Revisions to the classification, nomenclature, and diversity of eukaryotes. J. Eukaryot. Microbiol. 66, 4-119 (2019).

43. Katoh, K. \& Standley, D. M. MAFFT multiple sequence alignment software version 7: improvements in performance and usability. Mol. Biol. Evol. 30, 772-780 (2013).

44. Capella-Gutiérrez, S., Silla-Martínez, J. M. \& Gabaldón, T. trimAl: a tool for automated alignment trimming in large-scale phylogenetic analyses. Bioinformatics 25, 19721973 (2009).

45. Nguyen, L.-T., Schmidt, H. A., von Haeseler, A. \& Minh, B. Q. IQ-TREE: a fast and effective stochastic algorithm for estimating maximum-likelihood phylogenies. Mol. Biol. Evol. 32, 268-274 (2015).

46. Le, S. Q., Dang, C. C. \& Gascuel, O. Modeling protein evolution with several amino acid replacement matrices depending on site rates. Mol. Biol. Evol. 29, 2921-2936 (2012). 47. Minh, B. Q., Nguyen, M. A. T. \& von Haeseler, A. Ultrafast approximation for phylogenetic bootstrap. Mol. Biol. Evol. 30, 1188-1195 (2013).

48. Price, M. N., Dehal, P. S. \& Arkin, A. P. FastTree 2 - approximately maximumlikelihood trees for large alignments. PLOS ONE 5, e9490 (2010).

49. Huerta-Cepas, J., Serra, F. \& Bork, P. ETE 3: reconstruction, analysis, and visualization of phylogenomic data. Mol. Biol. Evol. 33, 1635-1638 (2016).

50. Steinegger, M. et al. HH-suite3 for fast remote homology detection and deep protein 
annotation. BMC Bioinform. 20, 473 (2019).

51. Huerta-Cepas, J. et al. Fast genome-wide functional annotation through orthology assignment by eggNOG-mapper. Mol. Biol. Evol. 34, 2115-2122 (2017).

52. Buchfink, B., Xie, C. \& Huson, D. H. Fast and sensitive protein alignment using DIAMOND. Nat. Methods 12, 59-60 (2015).

53. Vosseberg, J. et al. Data for: Timing the origin of eukaryotic cellular complexity with ancient duplications. figshare https://doi.org/10.6084/m9.figshare.10069985.v3 (2020).

\section{Acknowledgements}

We thank K. S. Marakova and E. V. Koonin for sharing their KOG-to-COG protein clusters with us. We are grateful to T. J. P. van Dam, E. S. Deutekom and G. J. P. L. Kops for useful advice and discussions. This work is part of the research programme VICI with project number 016.160.638, which is (partly) financed by the Netherlands Organisation for Scientific Research (NWO). T.G. acknowledges support from the Spanish Ministry of Science and Innovation for grant PGC2018-099921-B-I00 and from the European Union's Horizon 2020 research and innovation programme under grant agreement ERC-2016-724173.

\section{Author contributions}

J.J.E.v.H., T.G. and B.S. conceived the study. J.V. and J.J.E.v.H. performed the research. J.V., J.J.E.v.H., T.G. and B.S. analysed and interpreted the results. M.M.H. performed the analysis on the human phylome. M.M.H. and A.v.V. aided in the development of the tree analysis pipeline. L.M.v.W. implemented the ScrollSaw-based method. J.V., J.J.E.v.H. and B.S. wrote the manuscript, which was edited and approved by all authors.

\section{Competing interests}


The authors declare no competing interests. 


\section{Figure legends}

\section{Fig. 1 | Characterisation of duplications during eukaryogenesis.}

a, Density plot showing the distribution of the number of Pfam domains in present-day prokaryotes (green) and eukaryotes (purple) in comparison with the acquisition, invention and LECA estimates obtained from phylogenetic trees (see inset). b, Number of acquisitions or inventions that gave rise to a particular number of LECA families, demonstrating the skewedness of duplications across protein families. c, Odds of duplication for LECA families according to KOG functional categories. $81 \%$ of pairwise comparisons were significantly different (Supplementary Fig. 1). The poorly characterised categories and functions of very few families (cell motility, extracellular structures and nuclear structure) are not depicted. d, Odds of duplication for LECA families according to cellular localisation. 54\% of pairwise comparisons were significantly different (Supplementary Fig. 2). c-d, Numbers on the right side indicate the number of LECA families and dashed lines indicate the odds of all LECA families in total.

\section{Fig. 2 | Contribution of different phylogenetic origins to duplications during eukaryogenesis.}

a, Duplication tendency as fraction of clades having undergone at least one duplication. b, Multiplication factors, defined as the number of LECA families divided by the number of acquisitions or inventions. These numbers are shown beside the corresponding bar. a, $\mathbf{b}$, Dashed lines indicate the duplication tendency and multiplication factor for all acquisitions and LECA families. The four (a) and three (b) pairwise comparisons that did not give a significant $P$ value ( $\chi^{2}$ contingency table test) are shown. Prokaryotic: unclear prokaryotic ancestry (could not be assigned to a domain or lower taxonomic level). 


\section{Fig. 3 | Timing of acquisitions and duplications from different phylogenetic origins during eukaryogenesis.}

Ridgeline plot showing the distribution of corrected stem or duplication lengths, depicted as the additive inverse of the log-transformed values. Consequently, longer branches have a smaller value and vice versa. For clarity, a peak of near-zero branch lengths is not shown (see Extended Data Fig. 6). Numbers indicate the number of acquisitions or duplications for which the branch lengths were included. Groups of stem and duplication lengths are ordered based on the median value. The tree illustrates how the stem and duplication lengths were calculated; the symbols and colour schemes are identical to Fig. 1a. The phylogenetic distances between the acquisition or duplication and LECA were normalised by dividing it by the median branch length between LECA and the eukaryotic terminal nodes. In case of duplications the shortest of the possible normalised paths was used. Pairwise comparisons that did not give a significant $\mathrm{P}$ value (Mann-Whitney $U$ test) are shown.

\section{Fig. 4 | Timing of duplications during eukaryogenesis according to function and localisation.}

$\mathbf{a}, \mathbf{b}$, Ridgeline plots showing the distribution of duplication lengths for different functional categories (a) and cellular localisations (b). To enable a comparison with the timing of acquisitions, the binomial-based 95\% confidence interval of the median of the Asgard archaeal (FECA) and alphaproteobacterial stem lengths (mitochondrion) are depicted in grey, indicating the divergence of eukaryotes from their Asgard archaea-related and Alphaproteobacteria-related ancestors, respectively. Groups are ordered based on the median value. For significant differences between groups, see Supplementary Fig. 7-8. 


\section{Methods}

In this study we inferred and analysed two different sets of phylogenetic trees. The first set ('Pfam-ScrollSaw') was used for the main analysis, whereas the second set ('KOG-to-COG clusters') was used to verify our method to infer duplications during eukaryogenesis. We also used a third, already existing set of gene trees ('human phylome') to validate the use of branch lengths in case of duplications. Below we describe how we created and analysed the main set of phylogenetic trees. The second and third sets of gene trees are described in the Supplementary Methods.

\section{Data}

We used 209 eukaryotic (predicted) proteomes from an in-house dataset that has been used and described before ${ }^{34}$. Prokaryotic proteomes $(3,457$ in total) were extracted from eggNOG 4.5 $5^{35}$. The prokaryotic dataset was supplemented with nine predicted proteomes from the recently described Asgard superphylum ${ }^{6}$.

\section{Pfam assignment}

We used hmmsearch (HMMER v3.1b2 ${ }^{36}$ ) with the Pfam 31.0 profile hidden Markov models (HMMs $)^{16}$ and the corresponding gathering thresholds to assess to which Pfam what part of each prokaryotic and eukaryotic sequence should be assigned. We opted for Pfam profile HMMs to collect homologous sequences because of their sensitivity to detect homology. The domains that were hit were extracted from the sequences based on the envelope coordinates. If a sequence had hits to multiple Pfams and these hits were overlapping for at least 15 amino acids only the best hit was used. If the same Pfam had multiple hits in the sequence due to an insertion relative to the model the different hits were artificially merged. Since the latter is 
more prone to errors for short models and short sequences contain less phylogenetic signal, profile HMMs shorter than fifty amino acids were not considered for further analysis.

\section{Reduction of sequences}

For each Pfam, the number of prokaryotic sequences was reduced with kClust v1.0 ${ }^{37}$ using a clustering threshold of 2.93 , which corresponds to a sequence identity of $60 \%$. We chose this threshold because we expect it to retain sufficient prokaryotic diversity while removing sequences from related species to keep the analysis computationally feasible. However, because of horizontal gene transfer (HGT), it will also remove sequences from more distantly related species in some cases.

The number of eukaryotic sequences was reduced with a novel method ${ }^{38}$ based on the ScrollSaw approach ${ }^{14}$. The idea behind ScrollSaw is that instead of selecting a species subset a priori, the slowest evolving sequences are selected. In that way the resolution of deep nodes in trees from expanded families is drastically improved. Although in the original paper ${ }^{14}$ the distances between sequences were calculated with a maximum likelihood method, we used the bit score in $\mathrm{BLAST}^{39}$ as a proxy to obtain genetic distances. For each Pfam an all species versus all species BLAST was performed. Because we were only interested in the best hit the max_target_seqs option was set to 1. Although this option has raised some attention recently ${ }^{40,41}$, we only used it as a proxy for evolutionary distance and our analysis would not be seriously impacted by this option given the overall small sizes of our databases. Subsequently, bidirectional best hits (BBHs) between sequences from different eukaryotic groups were identified. Eukaryotic species can be grouped into different 'supergroups', whose names and definitions have changed following new findings ${ }^{19,42}$. The species in our dataset are from the following six groups: Archaeplastida + Cryptista, SAR + Haptista, Discoba, Metamonada, Obazoa and Amoebozoa. For our main analysis we used BBHs between 
sequences from two groups, because that provided the best resolution ${ }^{38}$. Although the exact position of the root of the eukaryotic tree of life is uncertain ${ }^{19}$, a likely position is between Opimoda (Obazoa and Amoebozoa in our set) and Diphoda (other supergroups) ${ }^{18}$. Therefore, BBHs between Opimoda and Diphoda sequences were identified and the corresponding sequences were used for phylogenetic analysis.

To assess the impact of a different position of the eukaryotic root, we also identified BBHs between five groups, merging Metamonada and Discoba into Excavata, and four groups, in which Archaeplastida + Cryptista and SAR + Haptista were combined as Diaphoretickes and Obazoa and Amoebozoa were together as Amorphea (see 'Effect of the position of the eukaryotic root').

\section{Phylogenetic analysis}

Multiple sequence alignments were made with MAFFT v7.310 $0^{43}$ (auto option) and trimmed with trimAl v1.4.rev15 ${ }^{44}$ (gap threshold 10\%). Phylogenetic trees were inferred with IQTREE v1.6.4 ${ }^{45}\left(\mathrm{LG} 4 \mathrm{X}\right.$ model $^{46}, 1000$ ultrafast bootstraps $\left.{ }^{47}\right)$. If the consensus tree had a higher likelihood than the best tree from the search, the first was used for further analysis. Because inferring trees for PF00005 (ABC transporter), PF00072 (response regulator receiver domain), PF00528 (binding-protein-dependent transport system inner membrane component), PF02518 (histidine kinase-, DNA gyrase B-, and HSP90-like ATPase) and PF07690 (major facilitator superfamily) in this way was too computationally demanding, we used FastTree v2.1.10 ${ }^{48}$ with the LG model to construct trees for these Pfams. These Pfams were not considered for branch length analysis.

\section{Tree analyses}

Removal of interspersing prokaryotes 
Trees were analysed with an in-house ETE $3^{49}$ script. We examined whether the tree contained prokaryotic sequences that probably reflect recent HGT events and that might interfere with our analysis. Prokaryotic sequences from a single genus that were in between eukaryotic sequences were pruned from the tree. If there was only one prokaryotic sequence in the tree it was kept only if it was an Asgard archaeal sequence, because it has been reported that sometimes only a single sequenced Asgard archaeon contains a homologue to sequences otherwise only present in eukaryotes ${ }^{6}$. This was the case for 16 trees containing LECA families (see below), including RPL28/MAK16, Sec23/24, UFM1 and the C-terminal domain of tubulins, for which the Asgard archaeal origin has been shown before. Because another prokaryotic outgroup to root these trees was lacking, they were not used to calculate stem lengths (see 'Branch length analysis').

\section{Annotation of eukaryotic nodes}

For each eukaryotic clade the nodes were annotated as duplications prior to LECA, LECA nodes, post-LECA nodes or unclassified. Only clades that contained at least one LECA node were of interest. The node combining the eukaryotic clade with the rest of the tree (if present) was annotated as acquisition node.

For the annotation of nodes in trees the information from the eukaryotic sequences that were not in the BBHs were included, since the number of eukaryotic sequences in the trees had been reduced. To correctly assign in-paralogues we additionally performed an own species versus own species BLAST for each Pfam (max_target_seqs 2). The sequences belonging to a Pfam that were not in the tree were mapped onto their best hits in the tree according to the BLAST score.

In order to infer reliable duplication nodes in the tree, duplication consistency scores were calculated for all internal nodes starting from the root of a eukaryotic clade. This score is 
the overlap of species at both sides of a node divided by the total number of species at both sides, taking both sequences in the tree and assigned sequences (as described above) into account. If the duplication consistency score was at least 0.2 and both daughter nodes fulfilled the LECA criteria, this node was annotated as a duplication node. The first LECA criterion was that a node had to have both Opimoda and Diphoda tree sequences in the clade.

Secondly, to take care of post-LECA HGT events among eukaryotes and of tree uncertainties, the mean presence of a potential LECA family in the five different supergroups (Obazoa, Amoebozoa, SAR+Haptista, Archaeplastida+Cryptista, Excavata) had to be at least $15 \%$. If a node did not fulfil the LECA criteria it was annotated as a post-LECA node.

The abovementioned thresholds were chosen based on manual inspection of a selection of trees. Using different thresholds for duplication consistency $(0,0.1,0.2,0.3)$ and LECA coverage scores $(0,5,10,15,20$ and $25 \%)$ had a gradual impact on the absolute numbers and quality measures, such as the fraction of well-supported LECA and duplication nodes (Supplementary Table 3). This underlines that the reported results were not contingent on the specific set of thresholds chosen and that for most nodes the duplication consistency and LECA coverage was high.

After this first annotation round all LECA nodes in the trees were re-evaluated. If there were duplication nodes in both daughters, the node connecting these duplications had to be a duplication node as well even though its duplication consistency score was below the threshold. This was only the case for two nodes in total. If there were duplication nodes in only one daughter lineage, the LECA node was annotated as unclassified. It could reflect a duplication event or a tree artefact due to rogue taxa. If there were no duplication nodes in either daughter lineages, all LECA nodes in the daughter lineages of this LECA node were reannotated as post-LECA nodes. 


\section{Rooting eukaryote-only trees}

For trees with only eukaryotic sequences and trees for which all prokaryotic sequences had been removed, inferring the root poses a challenge. For these trees duplication and LECA nodes were called in unrooted mode. The distances between the LECA nodes were calculated and the tree was rooted in the middle of the LECA nodes that were furthest apart, resulting in an additional duplication node at this root. If there were no duplications found in this way, because there were less than two duplications in the tree, rooting was tried on each internal node. The node that fulfilled the duplication criteria and that maximised the species overlap was chosen. If none fulfilled the criteria, it was checked if the entire tree fulfilled the LECA criteria. For Pfams for which we could not infer a tree because there were only two or three sequences selected, we also checked if this Pfam in itself fulfilled the LECA criteria. These Pfams correspond to eukaryote-specific families that did not duplicate.

\section{Sister group identification}

For each eukaryotic clade in trees also containing prokaryotic sequences the sister group was identified in an unrooted mode. By doing so, the eukaryotic clade initially had two candidate sister groups. Eukaryotic sequences in a sister group, if present, were ignored, as they could reflect HGT events, contaminations, tree artefacts or true additional acquisitions. To infer the actual sister group it was first checked if one of the two candidate sister groups was more likely by checking if one of them consisted only of Asgard archaea, TACK archaea, Asgard plus TACK archaea, alphaproteobacteria, beta/gammaproteobacteria, or alpha/beta/gammaproteobacteria. If so, that clade was chosen as the actual sister group. If both sister groups had the same identity or if both groups had another identity than the ones described above, the tree was rooted on the farthest leaf from the eukaryotic clade. In many cases the last common ancestor of the taxa in the sister group was Bacteria, Archaea or 
cellular organisms ("LUCA") according to the NCBI taxonomy. Such wide taxonomic assignments likely reflect extensive HGT among distantly related prokaryotes. In these cases it was checked if one of the previously mentioned groups or otherwise a particular phylum or proteobacterial class comprised a majority of the prokaryotic taxa to get a more precise sister group classification.

We observed that in a substantial number of cases there was another eukaryotic clade with LECA nodes in the sister group of a eukaryotic clade. These cases could reflect a duplication and subsequent loss in prokaryotes but probably reflect tree artefacts. Therefore these clades were ignored for the branch length analysis. Acquisitions that were nested, i.e. they shared exactly the same prokaryotic sister group because one acquisition had in its sister clade only one prokaryotic clade and one or multiple other acquisitions, were merged for further analysis.

\section{Branch length analysis}

Multiple branch lengths were calculated in clades containing LECA nodes. For the stem length (sl) the distance to the acquisition node - the node uniting the eukaryotic clade and its prokaryotic sister - was calculated for each LECA node. This distance was divided by the median of the distances from the LECA node to the eukaryotic leaves (eukaryotic branch lengths (ebl)) to correct for rate differences between orthologous groups as done before ${ }^{10}$. In case of multiple possible paths due to duplications, the minimum of these distances was used as the sl, since it was closest to sl values from zero-duplication clades. To calculate the duplication length (dl) a similar approach was followed, using the duplication node instead of the acquisition node.

To investigate the impact of rates after duplication in both paralogue lineages within a family, we also calculated for all duplication nodes in Asgard archaea-derived families the 
minimal sl going through these duplications (Extended Data Fig. 5c, d). In this way, we obtained an sl value for each duplication, in addition to the aforementioned sl value for each acquisition. These values were also divided into duplications in families that had undergone a transition from homomers to heteromers (proteasome, Snf7, TRAPP, Vps36 and OST3/OST6) and the rest (Extended Data Fig. 5e).

\section{Combining eukaryote-only Pfam families with prokaryotic donations in their clan}

The classification of protein families into Pfams is not based on taxonomic levels. A Pfam present only in eukaryotes can therefore be the result of a duplication event instead of a bona fide invention. To distinguish these possible scenarios we used the Pfam clans, in which related Pfam families are combined. If there were only eukaryote-only Pfams in a clan based on our analysis, these Pfams were merged into one invention event. If there was only one Pfam with an acquisition from prokaryotes and for this Pfam there was only one acquisition, the eukaryote-only Pfams were combined with this acquisition. If there were multiple acquisitions in a clan, a profile-profile search with $\mathrm{HH}$-suite $3 \mathrm{v} 3.0 .3^{50}$ was performed to assign eukaryote-only Pfams to an acquisition. Per acquisition in a clan an alignment was made from the tree sequences in the corresponding eukaryotic clade with MAFFT L-INS-i v7.310 ${ }^{43}$. Profile HMMs were made of these alignments (hhmake -M 50) and they were combined in a database (ffindex_build). The eukaryote-only Pfam HHMs were searched against the acquisition HHM database per clan with hhsearch. Each Pfam was assigned to the acquisition that had the best score.

\section{Functional annotation}

Functional annotation of sequences was performed using emapper-1.0.3 $3^{51}$ based on eggNOG orthology data ${ }^{35}$. Sequence searches were performed using DIAMOND v0.8.22.84 ${ }^{52}$. 
The most common KOG functional category among the tree sequences of a LECA node was chosen as the function of the LECA node. If there was not one function most common, the node was annotated as $\mathrm{S}$ (function unknown). For the functional annotation of duplication nodes a Dollo parsimony approach was used. For this we checked if there was one single annotation shared between LECA nodes at both sides, ignoring unknown functions. If this was not the case but the parent duplication node (if present) had a function, this function was also used for the focal duplication node. The functional annotation of the prokaryotic sister group was performed the same way as for a LECA node. In the figures the names of most categories were shortened for increased readability: Translation (Translation, ribosomal structure and biogenesis), RNA processing (RNA processing and modification), Replication (Replication, recombination and repair), Chromatin (Chromatin structure and dynamics), Cell cycle (Cell cycle control, cell division, chromosome partitioning), Signal transduction (Signal transduction mechanisms), Cell wall/membrane (Cell wall/membrane/envelope biogenesis), Intracellular trafficking (Intracellular trafficking, secretion, and vesicular transport), Protein modification (Posttranslational modification, protein turnover, chaperones), Energy (Energy production and conversion), Carbohydrates (Carbohydrate transport and metabolism), Amino acids (Amino acid transport and metabolism), Nucleotides (Nucleotide transport and metabolism), Coenzymes (Coenzyme transport and metabolism), Lipids (Lipid transport and metabolism), Inorganic ions (Inorganic ion transport and metabolism), Secondary metabolites (Secondary metabolites biosynthesis, transport and catabolism).

The same approach was used to assign cellular components to LECA and duplication nodes, using a custom set of gene ontology terms: extracellular region (GO:0005576), cell wall (GO:0005618), cytosol (GO:0005829), cytoskeleton (GO:0005856), mitochondrion (GO:0005739), cilium (GO:0005929), plasma membrane (GO:0005886), endosome (GO:0005768), vacuole (GO:0005773), peroxisome (GO:0005777), cytoplasmic vesicle 
(GO:0031410), Golgi apparatus (GO:0005794), endoplasmic reticulum (GO:0005783), nuclear envelope (GO:0005635), nucleoplasm (GO:0005654), nuclear chromosome (GO:0000228) and nucleolus (GO:0005730).

\section{Predicting the number of genes in LECA}

We used a linear regression model to predict the number of genes in LECA based on the inferred number of Pfam domains in LECA. For this we used the number of sufficiently long Pfam domains (see 'Pfam assignment' above) and the number of protein-coding genes in the eukaryotes in our dataset. The assumptions of a normal distribution of gene values and equal variance at each Pfam domain value were reasonably met after log transformation. Based on the relationship between the number of Pfam domains and genes in present-day eukaryotes, the number of protein-coding genes in LECA was estimated.

\section{Effect of the position of the eukaryotic root}

The eukaryotic phylogeny and the position of its root are incorporated in our analysis at two points: in the ScrollSaw step during the identification of BBHs between eukaryotic taxa and in the LECA criteria in the tree analyses. For computational reasons we limited the analysis on the impact of the eukaryotic phylogeny on our results to the Pfams that were only present in eukaryotes. In addition to the Opimoda-Diphoda BBHs, we selected the sequences from BBHs between either five or four supergroups, as described before, and inferred phylogenetic trees. The three different sets of trees were analysed using all seven root possibilities, given the monophyly of Amorphea, Diaphoretickes, Discoba and Metamonada. To fulfil the LECA criteria a node had to contain tree sequences from both sides of the root and the mean presence of a potential LECA family in the four different groups had to be at least $15 \%$. 


\section{Statistical analysis}

Overrepresentations of functions and localisations in duplications, inventions and innovations, and overrepresentations of sister groups in duplications and duplication tendencies were tested by comparing odds ratios with Fisher's exact tests (only pairwise comparisons of functions for inventions and localisations for innovations due to small sample sizes) or $\chi^{2}$ contingency table tests (rest). Differences in branch lengths were assessed with a Kruskal-Wallis test, followed by Mann-Whitney $U$ tests upon a significant outcome of the Kruskal-Wallis test. Only one Kruskal-Wallis test did not give a significant result (Extended Data Fig. 2b). Differences between two groups were assessed with Mann-Whitney $U$ tests. All performed tests were twosided. In all cases of multiple comparisons, the $\mathrm{P}$ values were adjusted to control the false discovery rate.

The ridgeline plots were drawn with the ggridges v0.5.1 R package (https://github.com/wilkelab/ggridges).

\section{Data availability}

Fasta files, phylogenetic trees and their annotations are available in figshare with the identifier ${ }^{53}$ doi:10.6084/m9.figshare.10069985.

\section{Code availability}

The code used to annotate the phylogenetic trees can be accessed in Github (https://github.com/JulianVosseberg/feca2leca). 


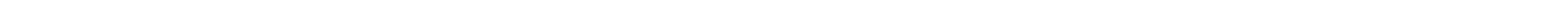




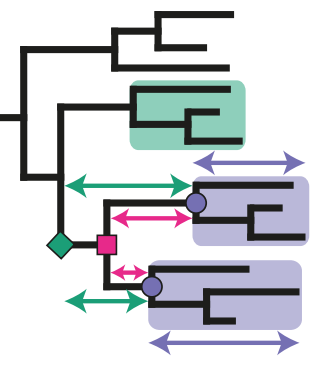

duplication length $(\mathrm{dl})=$

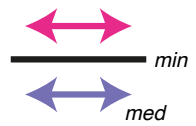

stem length $(\mathrm{sl})=$

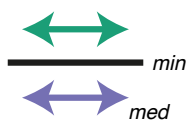

$P>0.05$
$\square \square$
$\square \square \square$
$\square \square$
$\square \square$
$\square \square \square$
$\square \square \square$

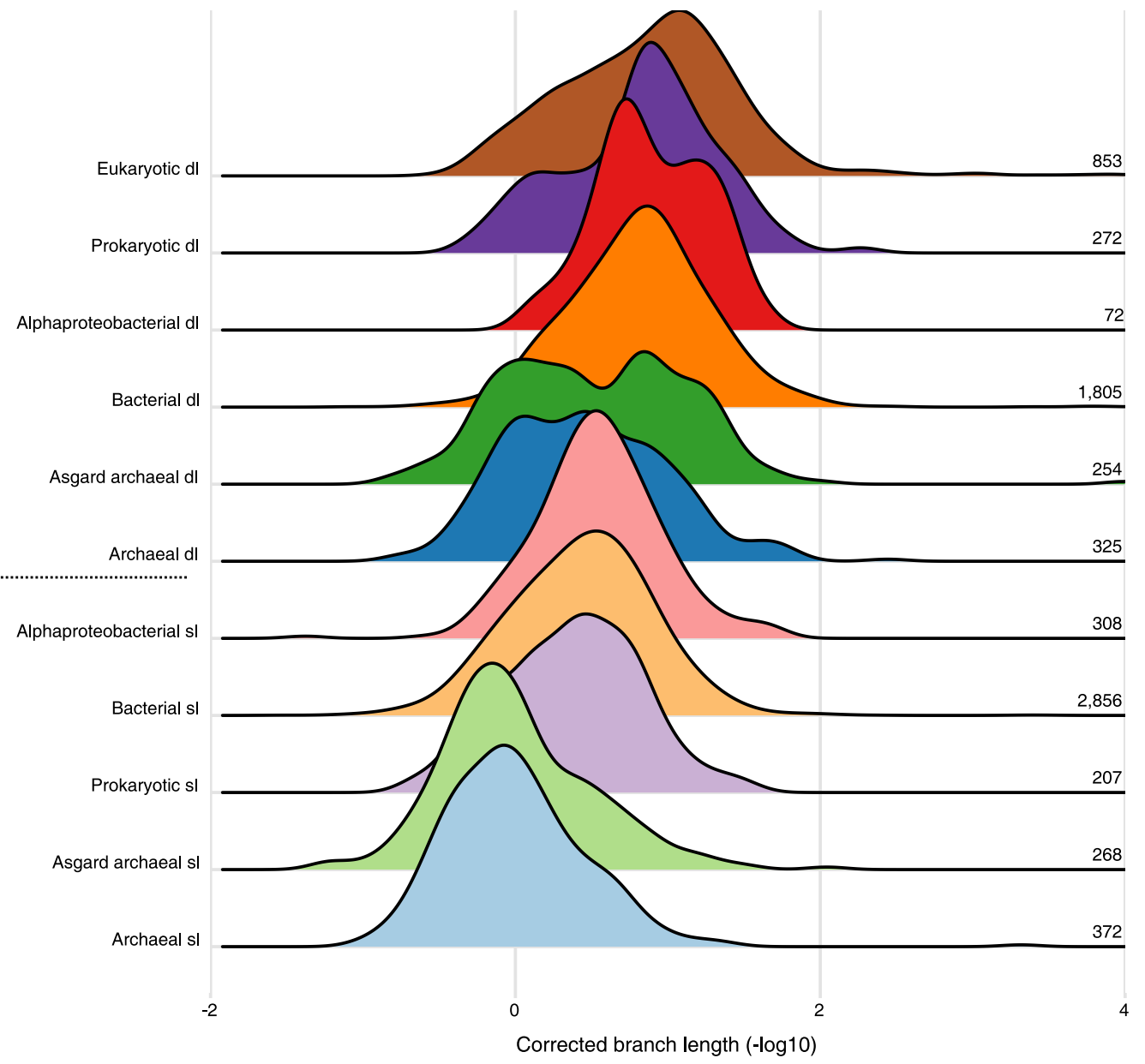


Inorganic ions

Signal transduction

Lipids

Transcription

Carbohydrates

Energy

Protein modification

Cell cycle Secondary metabolites

Replication

Amino acids

RNA processing

Intracellular trafficking

Translation

Cytoskeleton

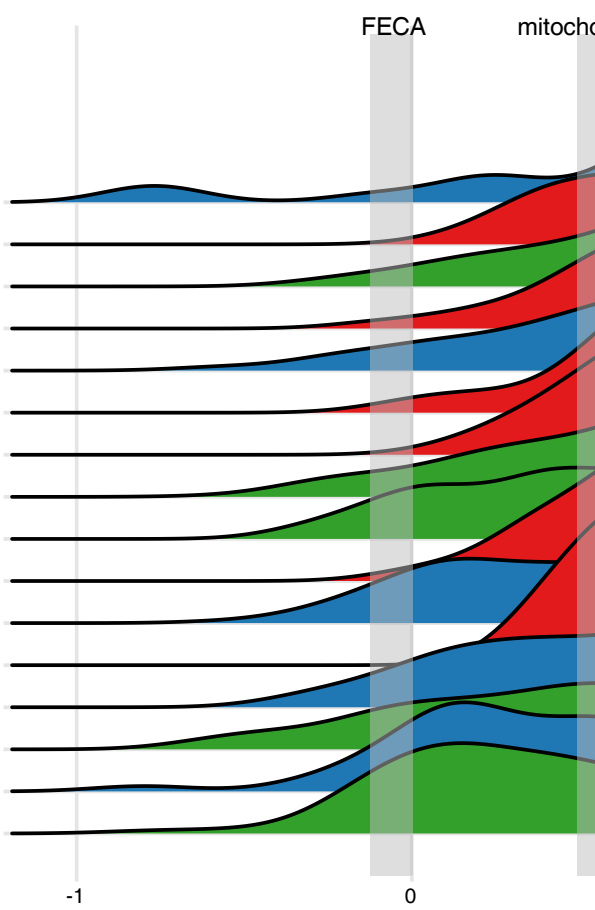

\section{ondrion}

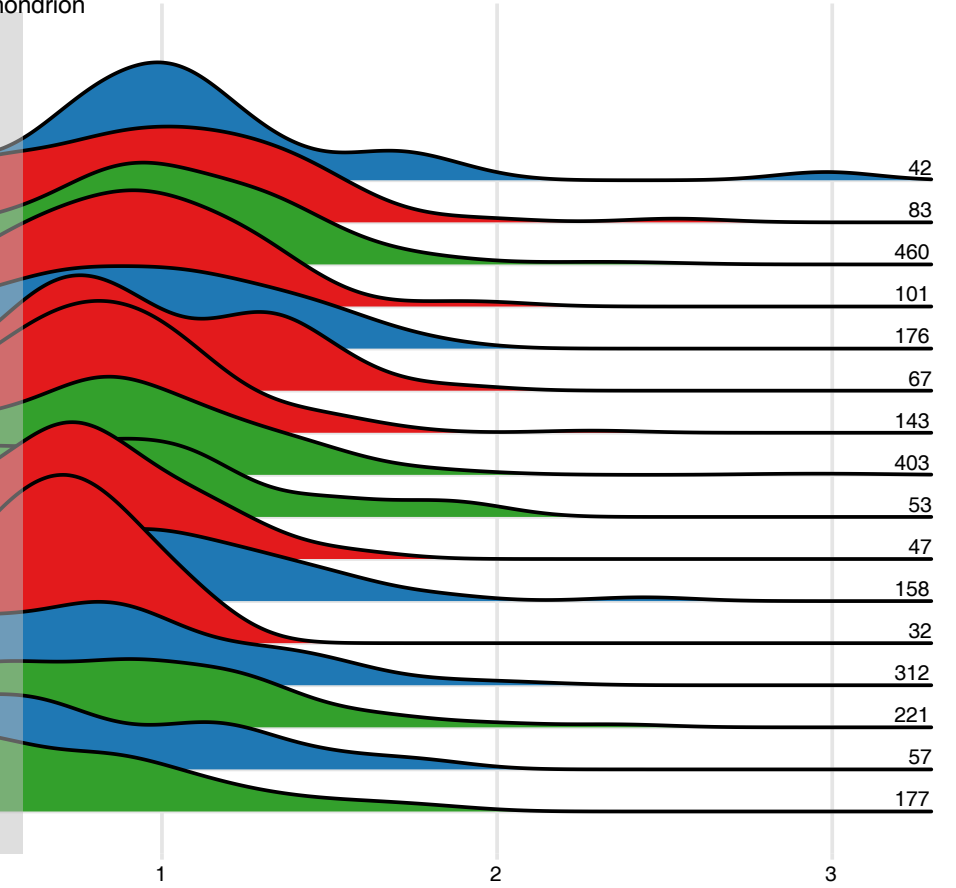

Corrected duplication length $(-\log 10)$

b

Enembrane system

Cytoskeleton

Nucleus

Other $\square$ Metabolic compartment

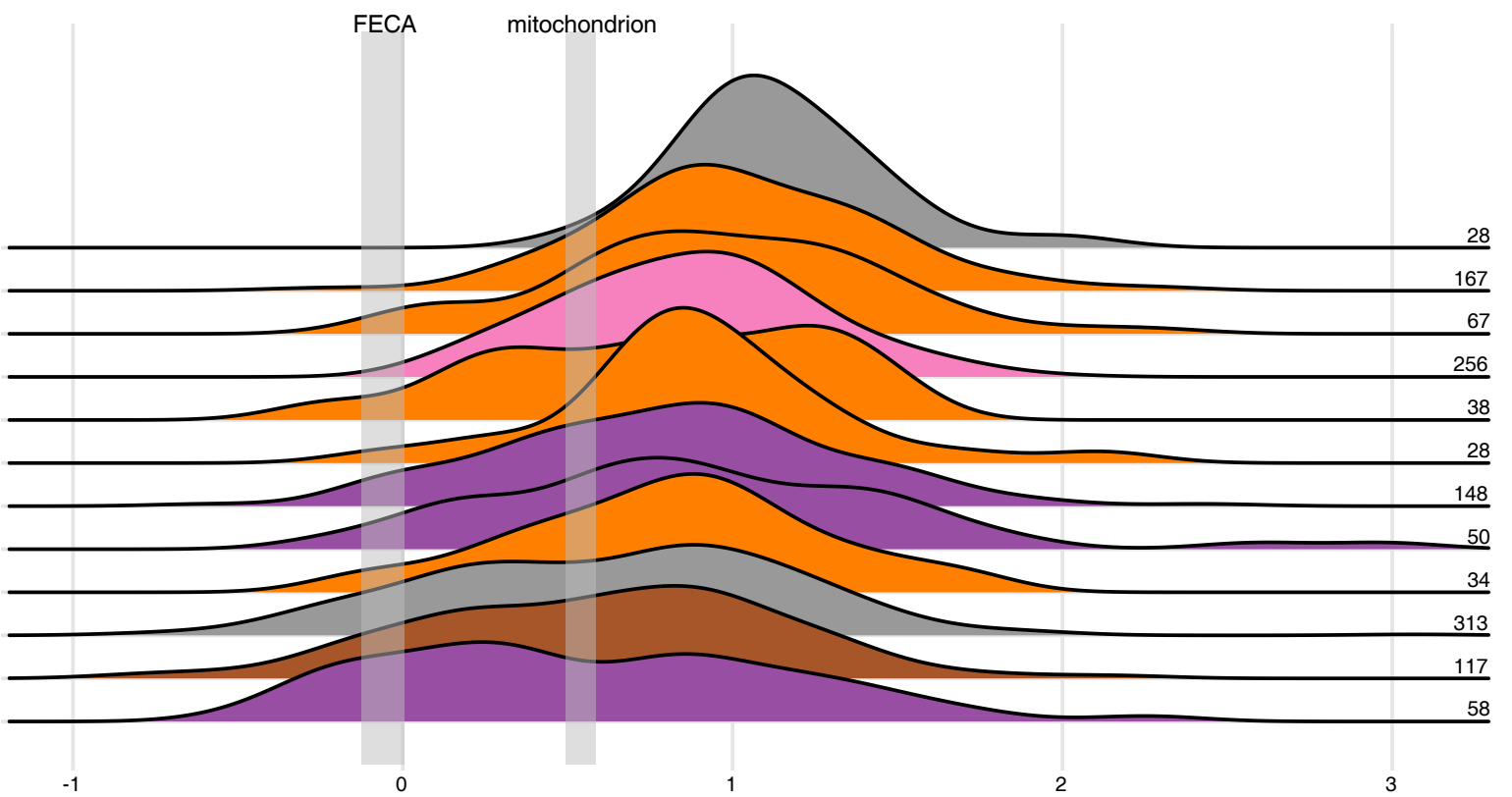

\section{mitochondrion}

Extracellular region

Plasma membrane

Endoplasmic reticulum

Mitochondrion

Golgi apparatus

Endosome

Nucleoplasm

Nuclear chromosome

Vacuole

Cytosol

Cytoskeleton

Nucleolus

Corrected duplication length $(-\log 10)$ 


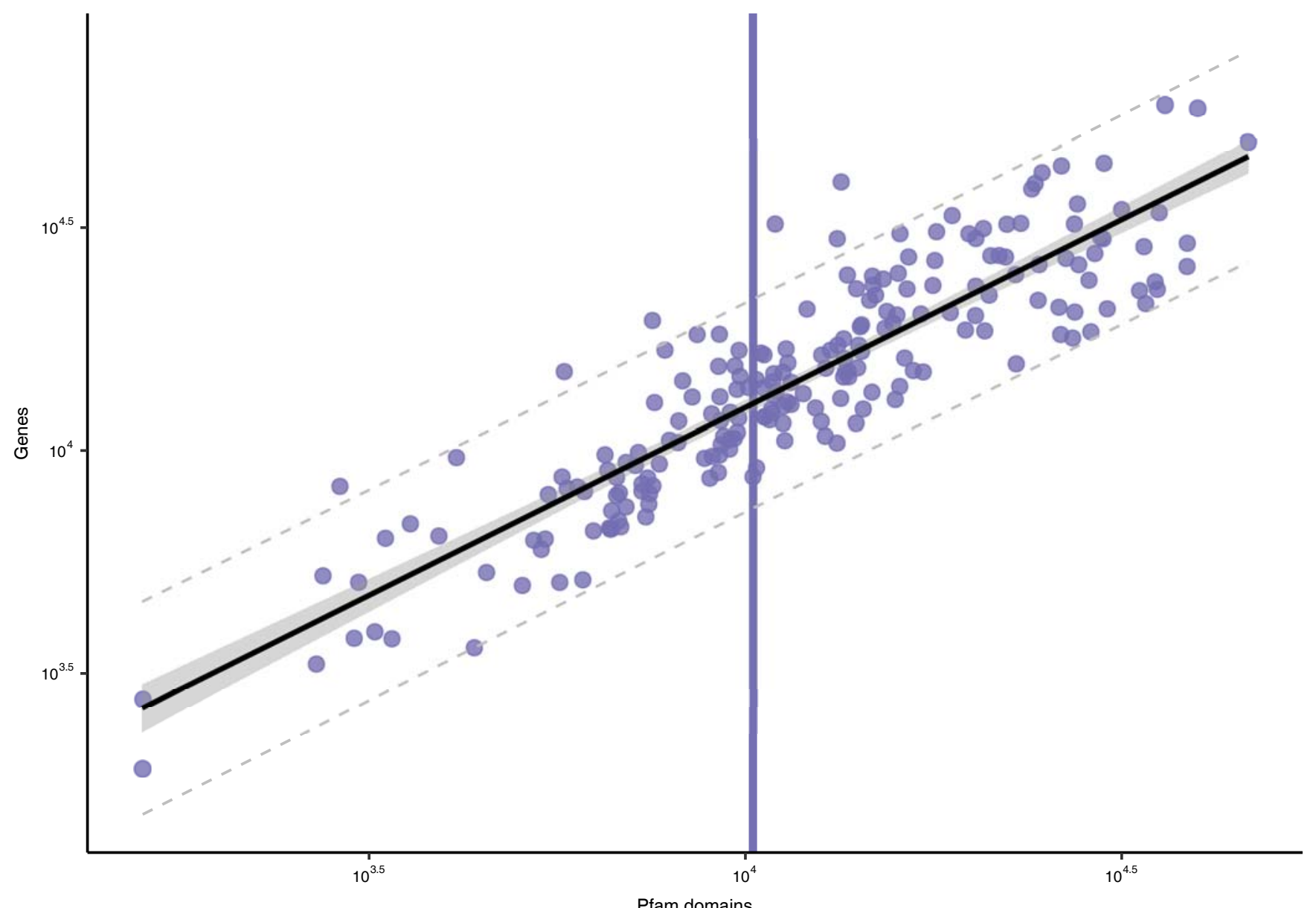

Pfam domains 

297

Asgard archaea

111 TACK archaea

14

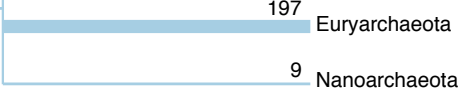

3

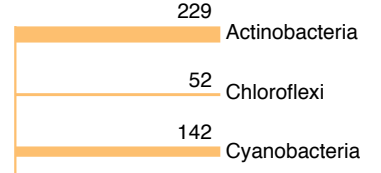

64

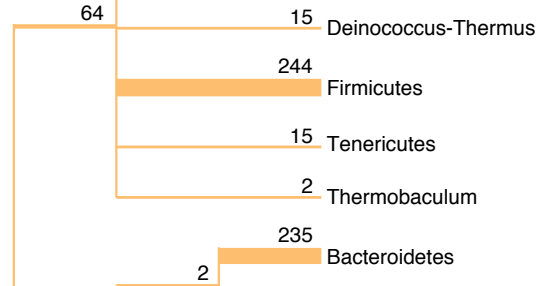

15 Chlorobi

10 Fibrobacteres

8 Gemmatimonadetes

41 Planctomycetes

60 Verrucomicrobia

8

44 Chlamydiae

16 Lentisphaerae

7 Elusimicrobia

64 Spirochaetes

5 Fusobacteria

10 Thermotogae

12 Aquificae

2 Chrysiogenetes

0 Dictyoglomi

3 Nitrospirae

7 Synergistetes

30 Acidobacteria

5 Deferribacteres

3 Thermodesulfobacteria

1 Haloplasma

198

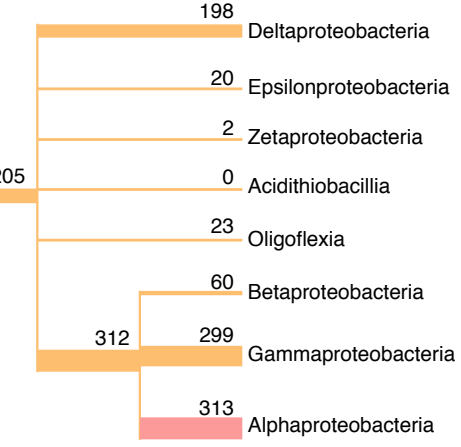

b

18

Candidatus Heimdallarchaeota archaeon AB_125

28

21

Candidatus Heimdallarchaeota archaeon LC_2

60

Candidatus Heimdallarchaeota archaeon LC_3

65

10

Lokiarchaeum sp. GC14_75

17

Candidatus Lokiarchaeota archaeon CR_4

20

Candidatus Odinarchaeota archaeon LCB_4

8 Candidatus Thorarchaeota archaeon AB_25

10

9 Candidatus Thorarchaeota archaeon SMTZ1-45

10 Candidatus Thorarchaeota archaeon SMTZ1-83

C

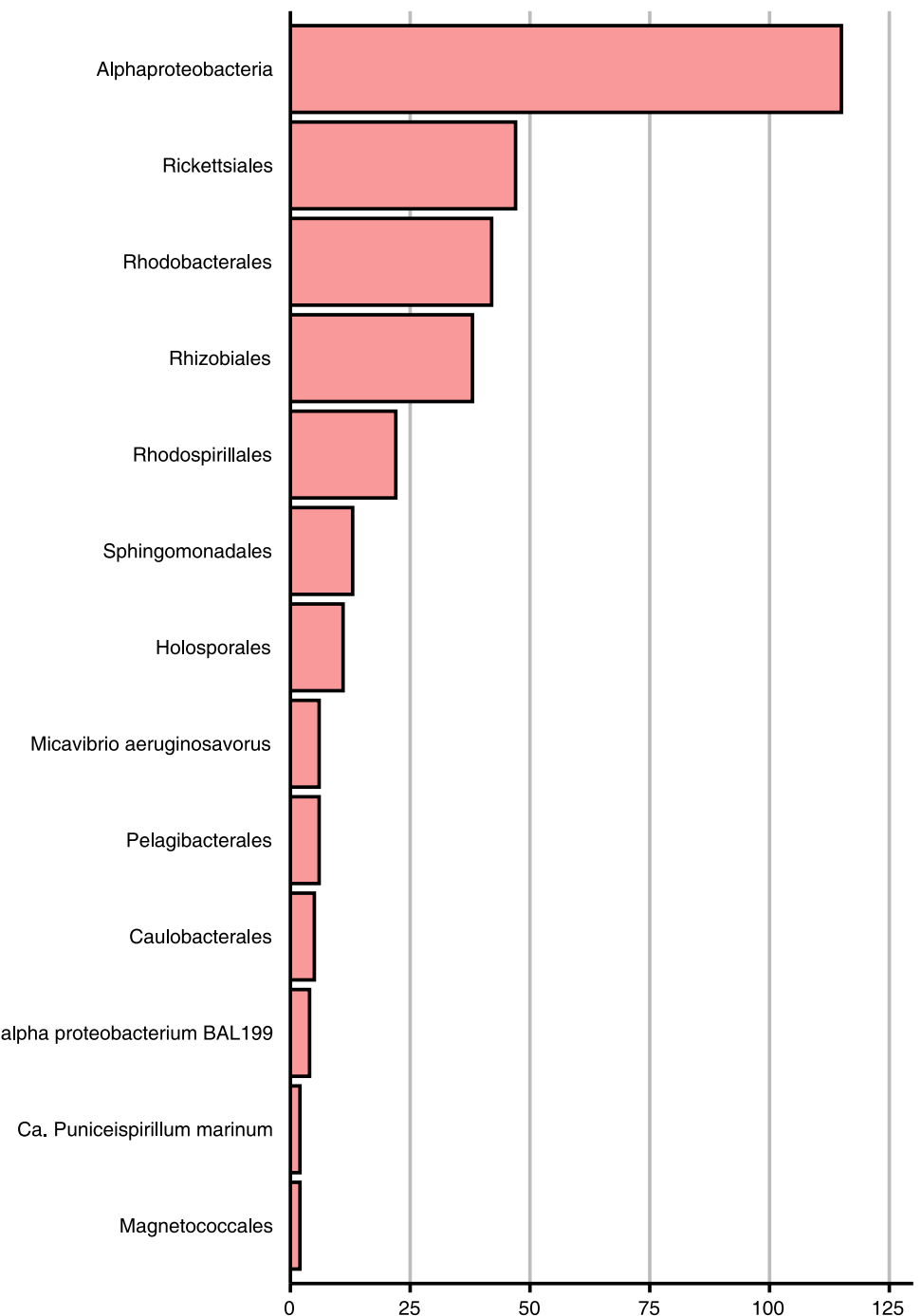

Acquisitions 


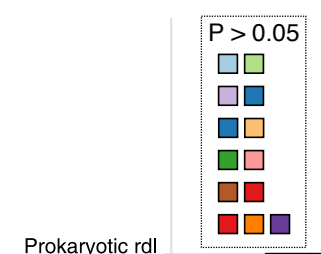

Alphaproteobacterial rdl Alphaproteobacterial rd

Eukaryotic rdl

Asgard archaeal rd

Archaeal rd

Alphaproteobacterial rs|

Bacterial rs

Prokaryotic rs $\mid$

Asgard archaeal rsl

Archaeal rsl
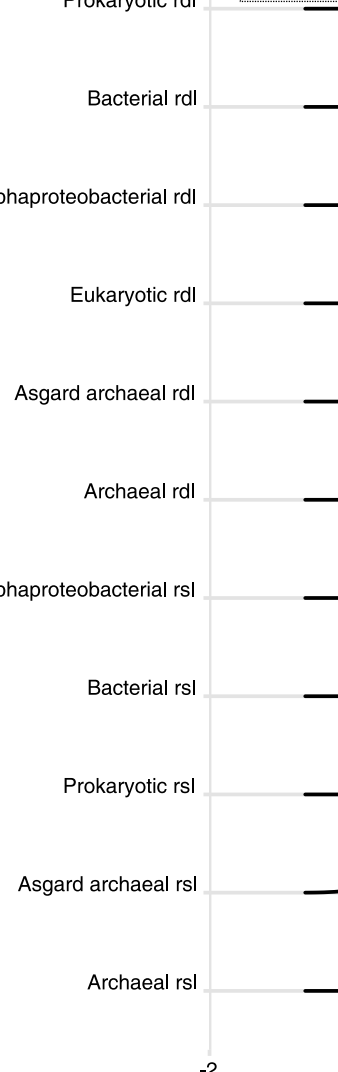

b

,

Alphaproteobacterial

0

2

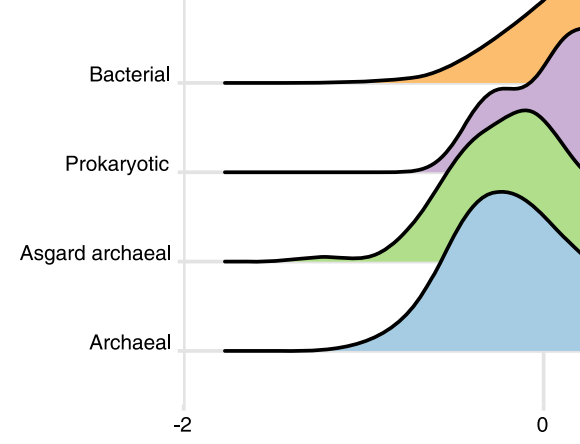

Raw branch length $(-\log 10)$

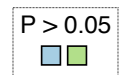

$\square \square$

Stem length $(-\log 10)$ 


\section{Supplementary information for:}

\section{Timing the origin of eukaryotic cellular complexity with ancient duplications}

Julian Vosseberg $^{1 *}$, Jolien J. E. van Hooff ${ }^{1 *}$, Marina Marcet-Houben ${ }^{2,3,4}$, Anne van Vlimmeren $^{1 \dagger}$, Leny M. van Wijk ${ }^{1}$, Toni Gabaldón ${ }^{2,3,4,5}$, Berend Snel ${ }^{1}$

${ }^{1}$ Theoretical Biology and Bioinformatics, Department of Biology, Faculty of Science, Utrecht University, Utrecht, The Netherlands

${ }^{2}$ Centre for Genomic Regulation, The Barcelona Institute of Science and Technology, Barcelona, Spain

${ }^{3}$ Life Sciences Department, Barcelona Supercomputing Center, Barcelona, Spain ${ }^{4}$ Mechanisms of Disease, Institute for Research in Biomedicine, Barcelona, Spain ${ }^{5}$ Institució Catalana de Recerca i Estudis Avançats, Barcelona, Spain

*These authors contributed equally to this work

${ }^{\S}$ Current affiliation: Ecologie Systématique Evolution, CNRS, Université Paris-Saclay, AgroParisTech, Orsay, France

${ }^{\dagger}$ Current affiliation: Department of Biological Sciences, Columbia University, New York City, United States of America

Correspondence to: toni.gabaldon@bsc.es (T.G.) or b.snel@uu.nl (B.S.) 
Table of contents

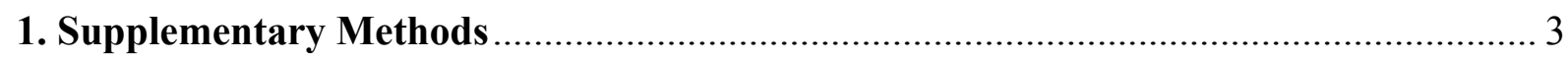

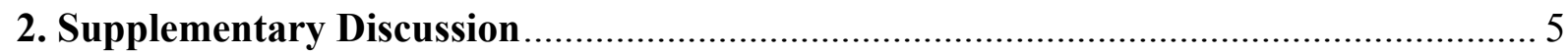

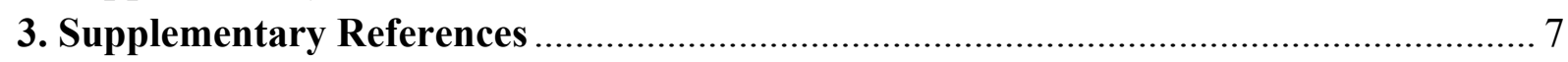

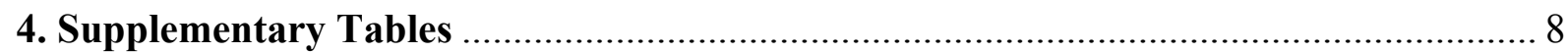

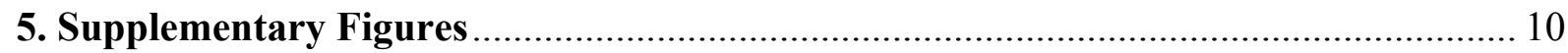




\section{Supplementary Methods}

\section{KOG-to-COG clusters analysis}

Selecting sequences and generating clusters

In order to compare our phylogenomics approach to previously reported accounts of duplications during eukaryogenesis, we applied it to the clusters of homologous sequences established by Makarova et al. ${ }^{11}$. Briefly, they mapped eukaryotic orthologous groups (KOGs) to homologous prokaryotic orthologous groups (COGs). In many cases, multiple KOGs mapped to a single COG, which often reflects a duplication during eukaryogenesis. Furthermore, KOGs had been clustered together if they are homologous to each other but lack a homologous COG. We used these KOG-to-COG clusters to assess if we, using a phylogenomics approach, were able to recapture the prevalence of gene duplications during eukaryogenesis that Makarova et al. observed by calculating ratios of KOGs to their affiliated COGs. Moreover, we took advantage of the current wealth of sequenced biodiversity by using an alternative, more representative species and sequence dataset compared to the original study. The results of this KOG-to-COG analysis can be found in Supplementary Table 1.

To recreate the KOG-to-COG clusters we used the COG assignment of the non-Asgard archaeal prokaryotic sequences provided by eggNOG and performed sequence profile searches with the Asgard archaeal and eukaryotic sequences. For the Asgard archaea, we downloaded profile HMMs of all COGs from eggNOG $4.5^{35}$ and assigned the Asgard protein sequences to COGs using hmmscan (HMMER v3.1b1 $1^{36}$ ). For eukaryotes, we selected ten species to obtain a good representation of eukaryotic diversity: Naegleria gruberi and Euglena gracilis (Excavata), Cladospihon okarmurans and Bigelowiella natans (SAR+Haptista), Guillardia theta and Klebsormidium flaccidum (Archaeplastida+Cryptista), Acanthamoeboa castellanii and Acytostelium subglobosum (Amoebozoa), and Capsaspora owczarzaki and Nuclearia sp. (Obazoa). We specifically opted for these species, because they were often involved in BBHs in the Pfam sequence selection (see Methods, 'Reduction of sequences'). Subsequently, we downloaded profile HMMs for orthologue clusters at the level of eukaryotes from eggNOG $4.5^{35}$. These contained both the supervised KOGs and nonsupervised orthologous groups (ENOGs). The original KOG-to-COG clusters from Makarova et al. ${ }^{11}$ did not include these ENOGs, but instead included candidate orthologous groups (TWOGs). Because these TWOGs are now obsolete, we sought to find the best matching ENOG based on the original sequence members of each TWOG. We combined the profile HMMs of these ENOGs with those of the KOGs and created a profile database. We performed hmmscan to assign protein sequences from the eukaryotic species to these KOGs/ENOGs.

Subsequently, for all KOGs/ENOGs and COGs, we reduced the number of sequences with kClust v1.0 ${ }^{37}$, using a score per column of 3.53 (approximately $70 \%$ sequence identity). We subsequently merged homologous sequences from eukaryotes, prokaryotes and Asgard archaea according to the KOG-to-COG mapping, resulting in updated KOG-to-COG clusters comprising sequences from diverse and informative eukaryotic and prokaryotic clades.

\section{Phylogenetic analyses}

For each KOG-to-COG cluster, we generated phylogenetic trees using an in-house pipeline also used previously ${ }^{10}$. The sequences were aligned using MAFFT v6.861 ${ }^{53}$, option -auto, and subsequently trimmed using trimAl v1.44 with a gap threshold of 0.1 . From these alignments, we constructed phylogenetic trees using FastTree v2.1.8 ${ }^{48}$ with WAG as evolutionary model. 


\section{Tree analyses}

For the annotation of nodes in KOG-to-COG trees a similar approach as for the PfamScrollSaw trees was followed. Only the criteria for LECA and duplication nodes were slightly different. Because of the lower number of eukaryotic species we here simply annotated a node as a LECA node if it contained both Opimoda and Diphoda sequences, and instead of a consistency score, we used a species overlap criterion of two to annotate duplication nodes: if the daughters both fulfilled the LECA criterion and shared at least two out of the in total ten eukaryotic species, it was annotated as a duplication node.

\section{Human phylome analysis}

To validate the use of branch lengths to time gene duplications, we also applied this approach to the numerous duplications in chordates. We inferred these from the human phylome, which we downloaded from PhylomeDB ${ }^{54}$ (Phylome ID 76: http://phylomedb.org/phylome_76). The results of this validation can be found in Extended Data Fig. $5 \mathrm{f}-\mathrm{h}$.

In this collection of phylogenetic trees we calculated the normalised vertebrate stem lengths by dividing the branch length between the common ancestors of chordates and vertebrates by the median branch length between the latter and present-day vertebrates. In case of duplications the stem length was included if the human seed protein was in the shortest possible stem length.

To obtain duplication lengths for duplications that occurred at different phylogenetic time points, we scanned in each tree the lineage of the human seed protein between the common ancestors of bilaterians and primates for the presence of duplications. Nodes connecting the seed with a human paralogue were annotated as duplication nodes. The phylogenetic time point ('age') of the duplication was obtained using the common ancestor of all species involved in the duplication event. Duplication lengths were calculated by dividing the branch length between the duplication node and the common ancestor of primates by the median branch length between the latter and present-day primates.

KOG functional categories were assigned to each protein in the phylome using emapper-2.0.1 $1^{51}$ based on eggNOG orthology data ${ }^{55}$. Functional annotation of the nodes in the trees were performed as described for duplication nodes before (see 'Functional annotation'). For each pair of duplications it was checked if they performed the same function and had the same age, performed the same function but had a different age or performed a different function but had the same age. For these pairs the difference in log-transformed duplication lengths was calculated. 


\section{Supplementary Discussion}

\section{Data sets used}

We tested two different data sets. The KOG-to-COG gene family clusters ${ }^{11}$ are a set specifically constructed to study duplications during eukaryogenesis and were therefore an ideal starting point. To get an even more complete picture of duplications we decided to use the Pfam database. By using this database we circumvented the need to use orthologous groups or infer homology. For certain families the Pfam domains correspond to full-length genes, whereas for others it is only a domain or even a motif. Although certain domain duplications are not fully independent of each other due to their presence in a single gene upon duplication, it is not unlikely that truly separated genes co-duplicated as well. Ideally, one would want to define the unit, either a domain or full-length gene, that evolved as an individual entity during eukaryogenesis. However, for various domains/genes it would be simply impossible to identify such a single entity, for example for domains that were independent upon acquisition or invention, but fused during eukaryogenesis and were therefore interdependent in LECA. This is especially probable given the abundance of gene fusion events during eukaryogenesis ${ }^{56}$.

\section{Sister group identity}

$7 \%$ of the acquisitions had an unclear prokaryotic ancestry. Both bacteria and archaea were present in the sister group with no phylum comprising a majority. A tentative explanation is that the identity of the donor is obscured due to post-acquisition HGT among distantly related prokaryotes. The tendency of these acquisitions to duplicate was similar to the Pfams with an archaeal ancestry (Fig. 2). This suggests that a large fraction of this group reflect genes present in the host lineage. Furthermore, a relatively large fraction of these acquisitions had another eukaryotic clade with LECA families in their sister group (34\%, between 3 and 10\% for the other groups), indicating that some of these acquisitions are placed in an incorrect, deep phylogenetic position. The stem and duplications lengths of these families with an unclear prokaryotic ancestry, however, were similar to those from families acquired from bacteria. Further research into these families is needed to elucidate their phylogenetic origin.

\section{Branch lengths analysis}

The stem lengths of acquisitions that happened simultaneously should approximate the same value, enabling us to assess the effect of duplications on branch lengths. Assuming the deep mitochondrial origin outside the alphaproteobacteria ${ }^{8}$, all acquisitions with alphaproteobacteria as sister group should correspond to the same event, namely the divergence of the pre-mitochondrial and alphaproteobacterial lineages. We observed a difference in stem lengths between duplicated and non-duplicated families from alphaproteobacterial origin, with duplicated families corresponding to longer stems (Extended Data Fig. 5a). Even using the shortest branch as stem, which we chose in case of duplications, could not fully account for the difference in stem lengths in these few duplicated families. In contrast, no difference in stem lengths with duplications was seen for acquisitions with an Asgard archaeal sister group (Extended Data Fig. 5b). We also looked at the effect of duplications on the stem lengths for the numerous duplications that occurred in the vertebrate stem. For these more recent duplications we observed a longer vertebrate stem in case of duplications (Extended Data Fig. 5f), in line with the alphaproteobacterial-related duplications. The presence of duplications can result in a subtle yet significant accelerated evolutionary rate in both daughter lineages.

Because we had detected more duplicated families with an Asgard archaeal sister group than an alphaproteobacterial one, we looked more in depth into the first. We could not detect 
a clear pattern of acceleration after duplications in both daughter lineages for different functional groups (Extended Data Fig. 5c-d). The barely significant difference for duplications related to cellular processes and signalling was dependent on the presence of outliers. Duplications that resulted in the transition from a homomer to a heteromer could have had a different effect on evolutionary rate as the selection pressures on the protein interface has changed. We did not observe a difference between duplications in families that underwent such a transition and other families (Extended Data Fig. 5e). However, the number of the first group was low and involved all duplications in these families, not only those resulting in the homomer-heteromer transition. Further research into these different effects of duplications is warranted. In conclusion, we could not confidently distinguish differences in rates for different groups of proteins upon duplication that could bias our results.

The inferred timing of acquisitions represent the earliest possibility of the actual acquisition, because they are the result of taxon sampling (i.e. which of the present-day organisms have been discovered, sequenced and/or included in the analysis) and historical contingency (i.e. which lineages have not gone extinct). Duplication nodes, on the other hand, represent the latest possibility of the actual acquisition, and therefore they could be used to attenuate the inferred acquisition time point.

\section{Comparison with Tria et al. ${ }^{20}$}

Our conclusions are in stark contrast with a recent preprint ${ }^{20}$, which reported remarkably fewer gene duplications and relatively many duplications in bacterial-related genes (compared to archaeal-related genes), which they interpret as being derived from the protomitochondrion. Based on their findings, the authors concluded that gene duplications support a eukaryogenesis model in which mitochondria entered early in eukaryogenesis, into a relatively simple, prokaryote-like host. We think this conclusion is insufficiently supported by their approach and resulting observations, because these have some clear deficits.

First and foremost, they infer very few eukaryogenesis duplications: 713 compared to 4,564 in our main dataset (see Supplementary Table 1). As an illustration: they did not recover well-documented greatly expanded protein families such as protein kinases and small GTPases $^{12,14}$, which we were able to recover (see Supplementary Table 2). The family that according to this preprint was most duplicated during eukaryogenesis was the dynein light chain family with 12 duplications.

Second, because they only inferred gene trees for eukaryotic sequences, they could not distinguish between duplications that happened during eukaryogenesis, those that happened before and pseudoparalogues (e.g., cytosolic and mitochondrial ribosomal proteins). Moreover, their limited usage of gene phylogenies also prohibits them from specifying the potential identity of the prokaryotic donor lineage.

Third, they do not discriminate between genes with alphaproteobacterial and another bacterial origin, but instead label all eukaryotic genes with bacterial affiliations as coming from the mitochondrial endosymbiont. Some, if not most, of these genes might in fact have been acquired through HGT from other bacterial lineages. Potentially, mixing these contributes to the relatively high number of gene duplications that count for endosymbiontderived genes.

Fourth, they did not include the Asgard archaea in their analysis, which are crucial for any inference about eukaryogenesis. This might explain why the duplications in the cytoskeletal and ubiquitin systems were not correctly identified as duplications associated to archaeal acquisitions ${ }^{5,6}$ in their analysis. This may have led to an underestimation of the duplications in host-related genes. 


\section{Supplementary References}

53. Katoh, K. \& Toh, H. Recent developments in the MAFFT multiple sequence alignment program. Brief. Bioinform. 9, 286-298 (2008).

54. Huerta-Cepas, J., Capella-Gutiérrez, S., Pryszcz, L. P., Marcet-Houben, M. \& Gabaldón, T. PhylomeDB v4: zooming into the plurality of evolutionary histories of a genome. Nucleic Acids Res. 42, D897-D902 (2014).

55. Huerta-Cepas, J. et al. eggNOG 5.0: a hierarchical, functionally and phylogenetically annotated orthology resource based on 5090 organisms and 2502 viruses. Nucleic Acids Res. 47, D309-D314 (2019).

56. Méheust, R. et al. Formation of chimeric genes with essential functions at the origin of eukaryotes. BMC Biol. 16, 30 (2018). 


\section{Supplementary Tables}

Supplementary Table 1. Comparison of different datasets.

\begin{tabular}{lrrr}
\hline & Pfam-ScrollSaw trees & $\begin{array}{r}\text { Trees from recreated } \\
\text { KOG-to-COG clusters }\end{array}$ & $\begin{array}{r}\text { Original KOG-to-COG } \\
\text { clusters (no trees) })^{11}\end{array}$ \\
\hline Acquisitions & 4,335 & 3,460 & 1,092 \\
Inventions & 1,334 & 883 & 1,058 \\
Duplications & 4,564 & 4,888 & 1,987 \\
LECA families & 10,233 & 9,231 & 4,137 \\
Multiplication factor & 1.81 & 2.12 & 1.92 \\
\hline
\end{tabular}

\section{Supplementary Table 2. Most expanded acquisitions or inventions during} eukaryogenesis.

\begin{tabular}{llr}
\hline Pfam & Ancestry & Number of LECA families \\
\hline Total & & 123 \\
\hline Mitochondrial carrier* & Invention & 106 \\
Protein kinase & Planctomycetes & 92 \\
RING-finger/U-box & Actinobacteria & 82 \\
PH domain & Haloplasma & 76 \\
Ubiquitin & Asgard archaea & 72 \\
C2 domain & Prokaryotes & 71 \\
RNA recognition motif & A $\beta$-proteobacteria & 66 \\
Tetratricopeptide repeat & Firmicutes & 50 \\
POZ domain & Chlamydiae & 46 \\
FYVE/PHD zinc finger & Invention & 76 \\
\hline Asgard archaea & & 19 \\
\hline Ubiquitin & Asgard archaea & 19 \\
Vps51 domain superfamily & Asgard archaea & 16 \\
Cyclin & Asgard archaea & 15 \\
Helix-turn-helix & Asgard archaea & 11 \\
Thioredoxin & Asgard archaea & 10 \\
Helix-turn-helix & Asgard archaea & 10 \\
Golgi-transport & Asgard archaea & 10 \\
Helix-turn-helix & Asgard archaea & 10 \\
Gelsolin repeat & Asgard archaea & 10 \\
Gelsolin repeat & Asgard archaea & 9 \\
\hline Alphaproteobacteria & & 8 \\
\hline Sterile alpha motif & Alphaproteobacteria & 5 \\
Galactosyltransferase & Alphaproteobacteria & 5 \\
EF-hand 8 & Alphaproteobacteria & 5 \\
Iron/zinc purple acid phosphatase-like protein C & Alphaproteobacteria & 5 \\
DDE superfamily endonuclease & Alphaproteobacteria & 4 \\
ABC transporter & Alphaproteobacteria & \\
Alpha/beta hydrolase fold & Alphaproteobacteria & \\
Ferric reductase & Alphaproteobacteria & \\
\hline
\end{tabular}

*A mitochondrial carrier protein typically contains three of these domains. 
Supplementary Table 3. Effect of different duplication consistency and LECA coverage thresholds.

\begin{tabular}{|c|c|c|c|c|c|c|}
\hline $\begin{array}{r}\text { Duplication } \\
\text { consistency } \\
\text { score }\end{array}$ & $\begin{array}{r}\text { LECA coverage } \\
\text { score }\end{array}$ & $\begin{array}{r}\text { Number of } \\
\text { LECA families }\end{array}$ & $\begin{array}{r}\text { Number of } \\
\text { unclassified } \\
\text { nodes }\end{array}$ & $\begin{array}{r}\text { Number of } \\
\text { eukaryotic } \\
\text { clades without } \\
\text { LECA families }\end{array}$ & $\begin{array}{l}\text { Fraction well- } \\
\text { supported* } \\
\text { LECA nodes }\end{array}$ & $\begin{array}{r}\text { Fraction well- } \\
\text { supported } \\
\text { duplication } \\
\text { nodes }\end{array}$ \\
\hline \multirow[t]{6}{*}{$\mathbf{0}$} & $\mathbf{0}$ & 23,567 & 5,304 & 19,661 & 0.47 & 0.26 \\
\hline & 5 & 19,724 & 4,801 & 21,556 & 0.43 & 0.26 \\
\hline & 10 & 15,671 & 4,013 & 23,095 & 0.41 & 0.27 \\
\hline & 15 & 12,531 & 3,205 & 24,314 & 0.42 & 0.28 \\
\hline & 20 & 10,248 & 2,591 & 25,145 & 0.43 & 0.29 \\
\hline & 25 & 8,648 & 2,000 & 25,731 & 0.45 & 0.30 \\
\hline \multirow[t]{6}{*}{10} & $\mathbf{0}$ & 18,588 & 2,928 & 19,661 & 0.53 & 0.24 \\
\hline & 5 & 16,028 & 3,221 & 21,556 & 0.51 & 0.24 \\
\hline & 10 & 13,317 & 2,522 & 23,095 & 0.49 & 0.26 \\
\hline & 15 & 11,048 & 2,137 & 24,314 & 0.50 & 0.26 \\
\hline & 20 & 9,339 & 1,916 & 25,145 & 0.51 & 0.28 \\
\hline & 25 & 8,083 & 1,651 & 25,731 & 0.52 & 0.28 \\
\hline \multirow[t]{6}{*}{20} & $\mathbf{0}$ & 16,547 & 2,354 & 19,661 & 0.55 & 0.24 \\
\hline & 5 & 14,335 & 2,514 & 21,556 & 0.53 & 0.24 \\
\hline & 10 & 12,092 & 2,029 & 23,095 & 0.52 & 0.25 \\
\hline & 15 & 10,233 & 1,772 & 24,314 & 0.52 & 0.26 \\
\hline & 20 & 8,821 & 1,586 & 25,145 & 0.53 & 0.27 \\
\hline & 25 & 7,764 & 1,397 & 25,731 & 0.54 & 0.28 \\
\hline \multirow[t]{6}{*}{30} & $\mathbf{0}$ & 15,241 & 1,976 & 19,661 & 0.56 & 0.25 \\
\hline & 5 & 13,161 & 1,924 & 21,556 & 0.54 & 0.25 \\
\hline & 10 & 11,147 & 1,673 & 23,095 & 0.54 & 0.26 \\
\hline & 15 & 9,523 & 1,490 & 24,314 & 0.54 & 0.27 \\
\hline & 20 & 8,306 & 1,360 & 25,145 & 0.55 & 0.28 \\
\hline & 25 & 7,420 & 1,235 & 25,731 & 0.55 & 0.29 \\
\hline
\end{tabular}

*Ultrafast bootstrap support value 95 or higher. 


\section{Supplementary Figures}

a

Amino acids

Nucleotides

Translation

Carbohydrates

Cell wall/membrane

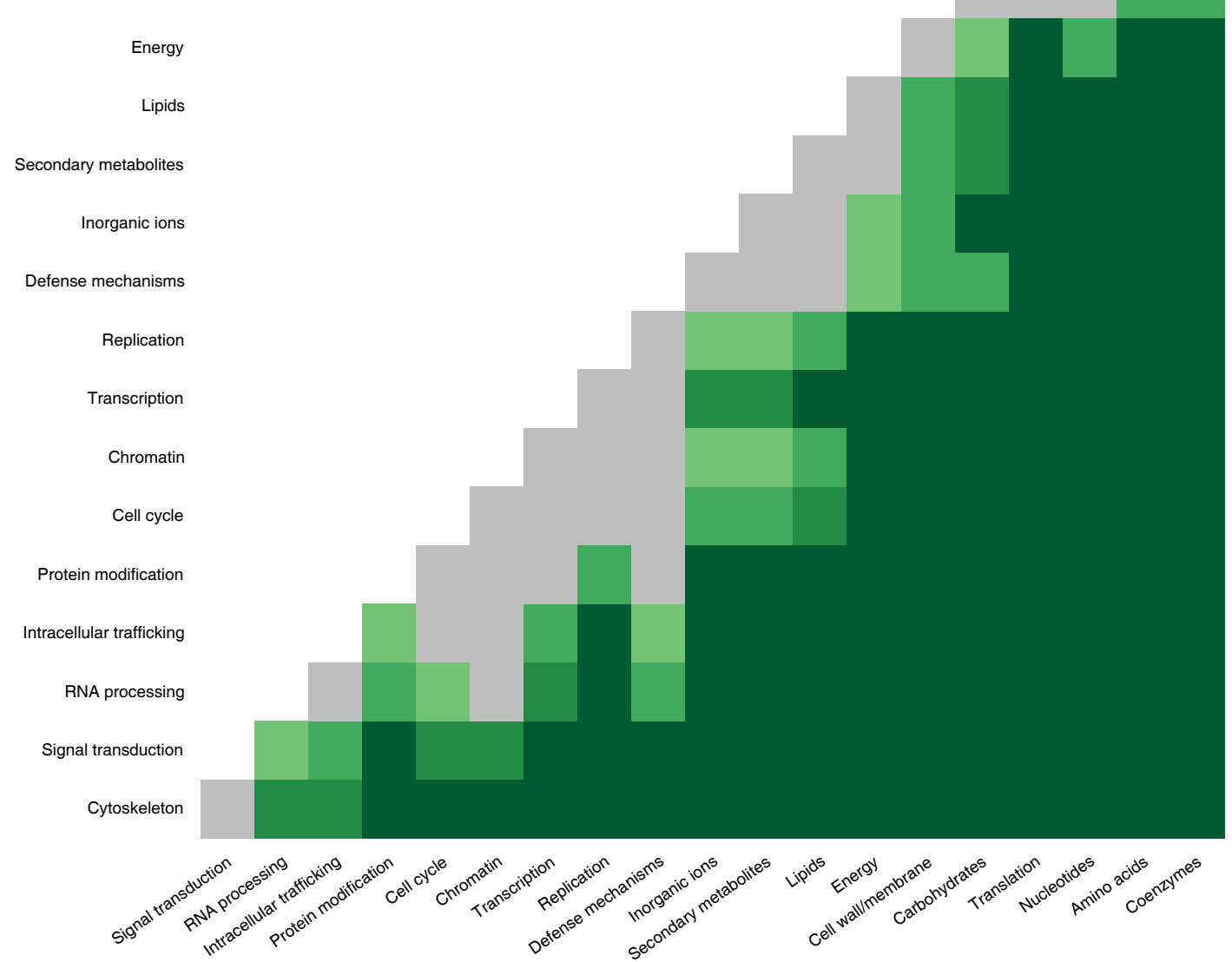

b

Information storage and processing

Cellular processes and signalling

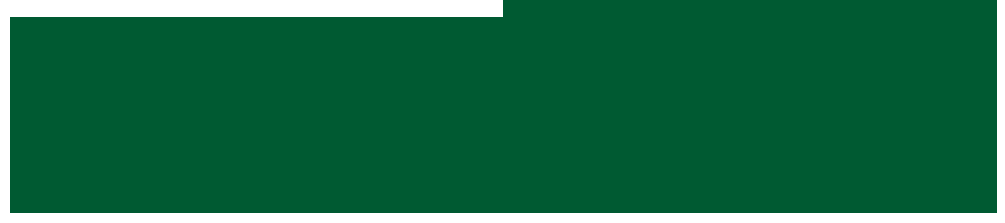

Information storage and processing

Metabolism

$P<0.0001-P<0.001$
$P$

Supplementary Fig. 1 | Contribution of duplications to families with a particular function.

Statistical significance of pairwise comparisons ( $\chi^{2}$ contingency table tests) between the proportions of LECA families being derived from duplications for different functional categories (a) and the corresponding broad categories (b). The values for each functional category are shown in Fig. 1c. The axis labels are ordered based on the odds of duplication. 
Extracellular region

Vacuole

Nuclear chromosome

Nucleoplasm

Nuclear envelope

Golgi apparatus

Plasma membrane

Cytoplasmic vesicle
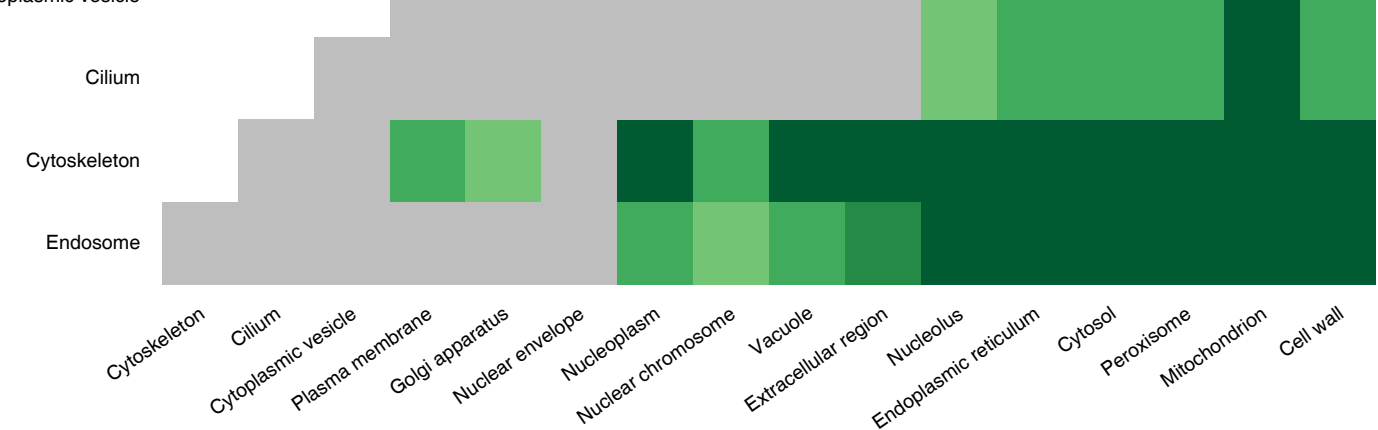

b

Other

Nucleus

Endomembrane system

Cytoskeleton

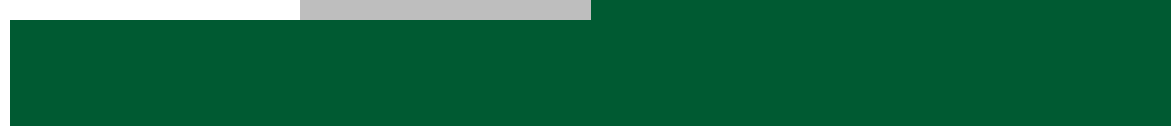

Endomembrane system

Nucleus

Other

Metabolic compartment

$\mathrm{P}<0.0001$

$P<0.001$

$P<0.01$

$\mathrm{P}<0.05$

$P \geq 0.05$

Supplementary Fig. 2 | Contribution of duplications to families with a particular cellular localisation.

Statistical significance of pairwise comparisons ( $\chi^{2}$ contingency table tests) between the proportions of LECA families being derived from duplications for different localisations (a) and the corresponding broad categories (b). The values for each localisation are shown in Fig. $1 \mathrm{~d}$. The axis labels are ordered based on the odds of duplication. 
Translation

Protein modification

Replication

Defense mechanisms

Signal transduction

RNA processing
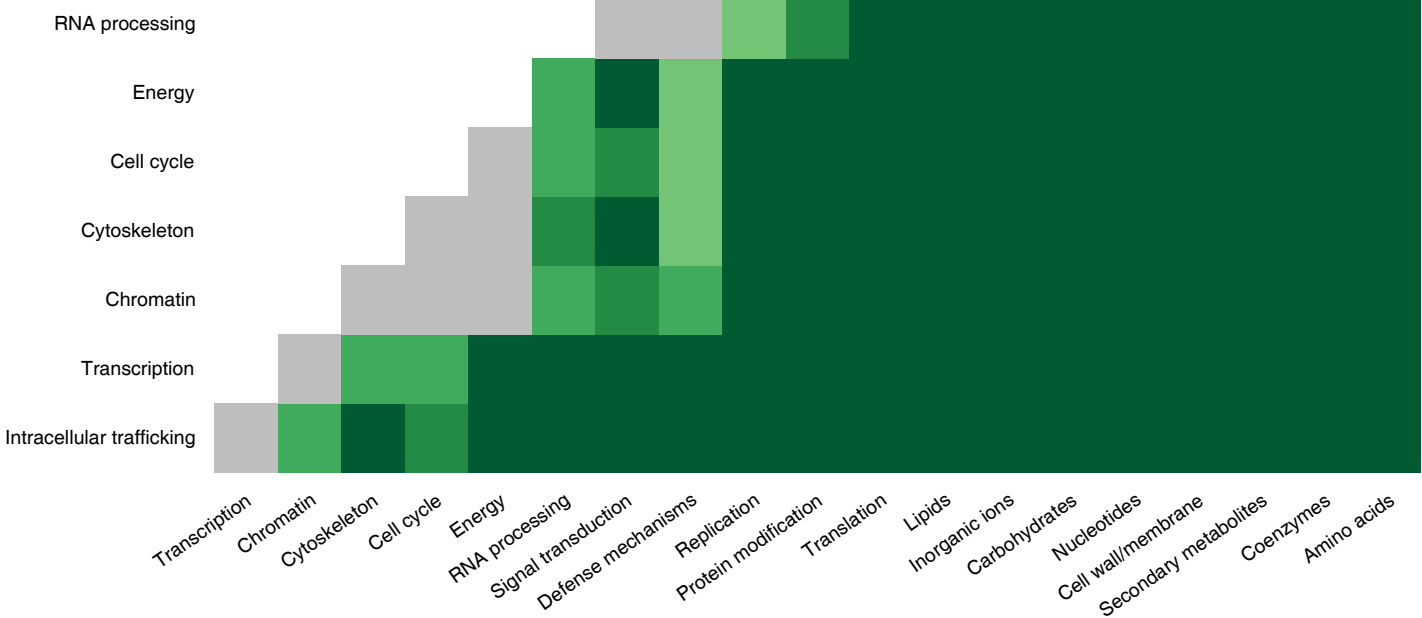

b

Information storage and processing

Cellular processes and signalling

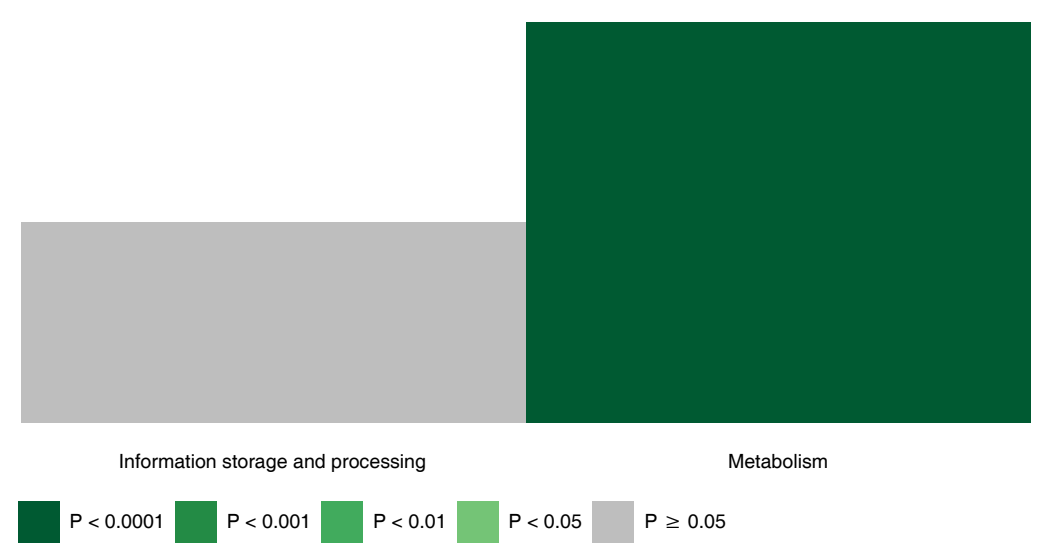

Supplementary Fig. 3 | Contribution of inventions to families with a particular function.

Statistical significance of pairwise comparisons (Fisher's exact tests) between the proportions of LECA families being derived from inventions for different functional categories (a) and the corresponding broad categories (b). The values for each functional category are shown in Extended Data Fig. 3a. The axis labels are ordered based on the invented fraction. 
a

Extracellular region

Plasma membrane

Peroxisome

Vacuole

Mitochondrion

Golgi apparatus

Endoplasmic reticulum

Nuclear chromosome

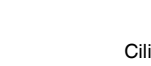

Cytoskeleton

Nucleolus

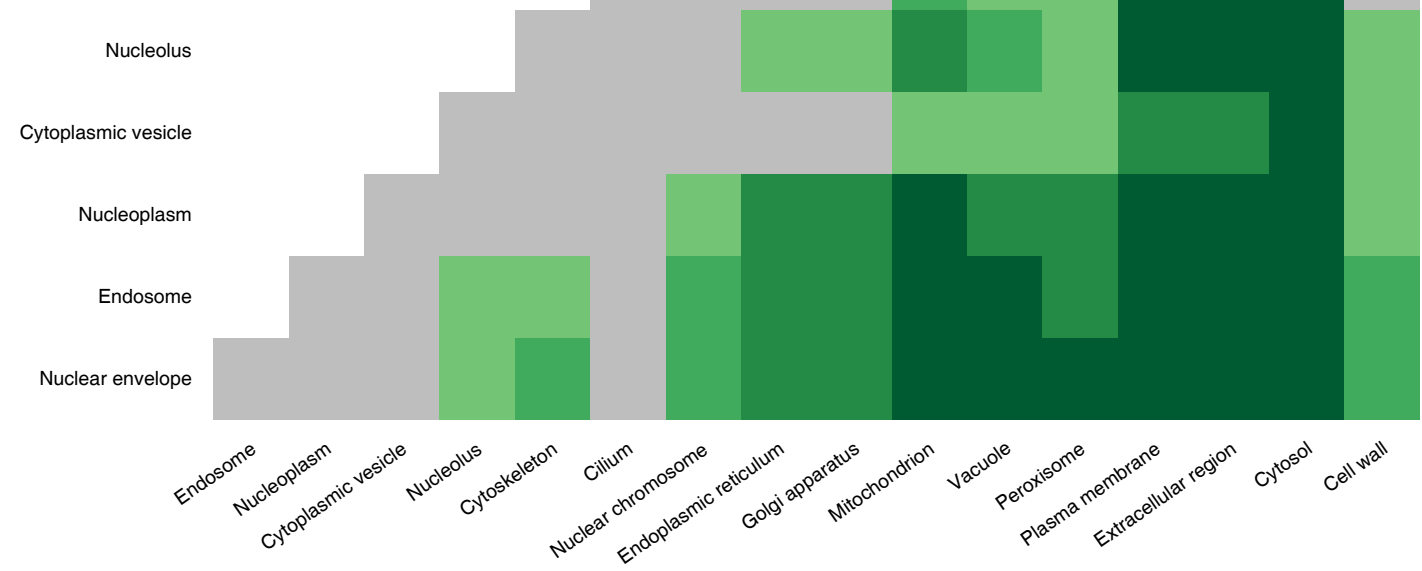

b

Metabolic compartment

Endomembrane system

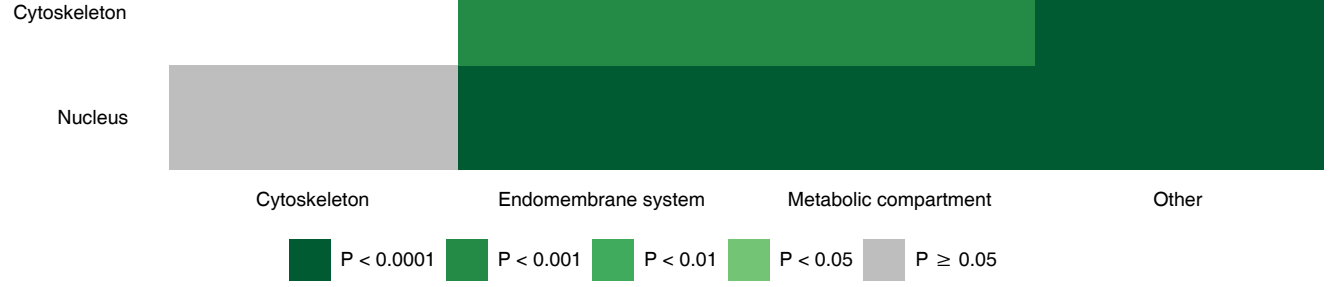

Supplementary Fig. 4 | Contribution of inventions to families with a particular cellular localisation.

Statistical significance of pairwise comparisons ( $\chi^{2}$ contingency table tests) between the proportions of LECA families being derived from inventions for different localisations (a) and the corresponding broad categories (b). The values for each localisation are shown in Extended Data Fig. 3c. The axis labels are ordered based on the invented fraction. 


\section{Chromatin \\ Cell cycle}

Signal transduction

RNA processing

Transcription

Intracellular trafficking

Cytoskeleton

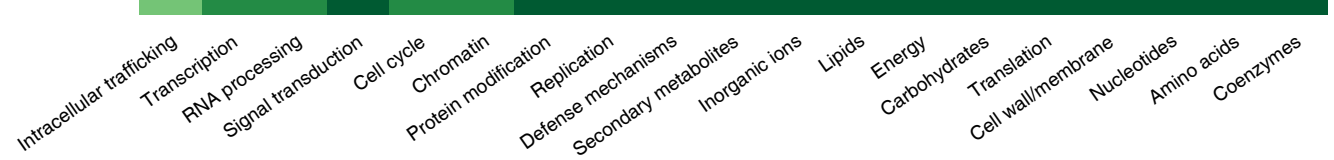

b

Information storage and processing

Cellular processes and signalling

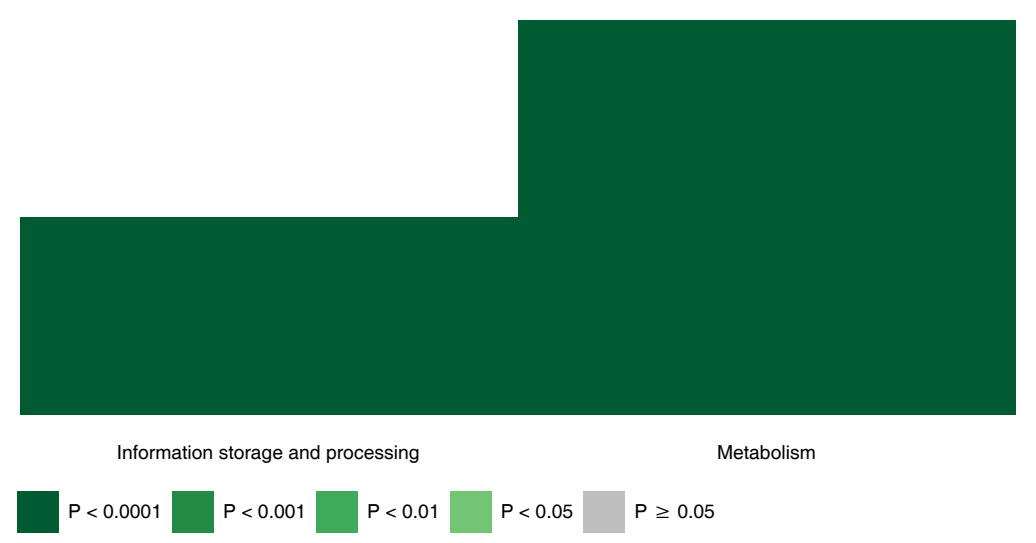

\section{Supplementary Fig. 5 | Contribution of innovations to families with a particular} function.

Statistical significance of pairwise comparisons ( $\chi^{2}$ contingency table tests) between the proportions of LECA families being derived from a eukaryotic innovation (invention or duplication) for different functions (a) and the corresponding broad categories (b). The values for each functional category are shown in Extended Data Fig. 3b. The axis labels are ordered based on the innovated fraction. 


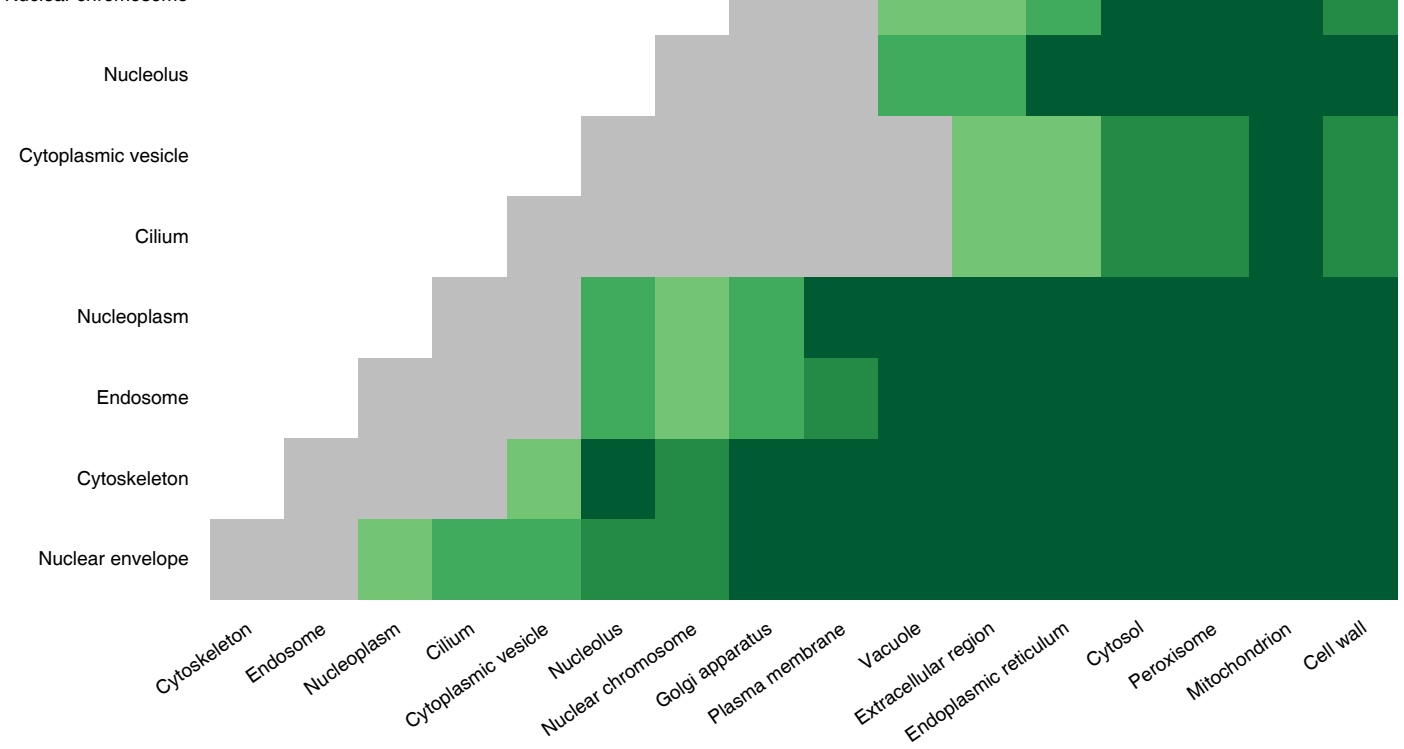

b

Other

Nucleus Cytoskeleton

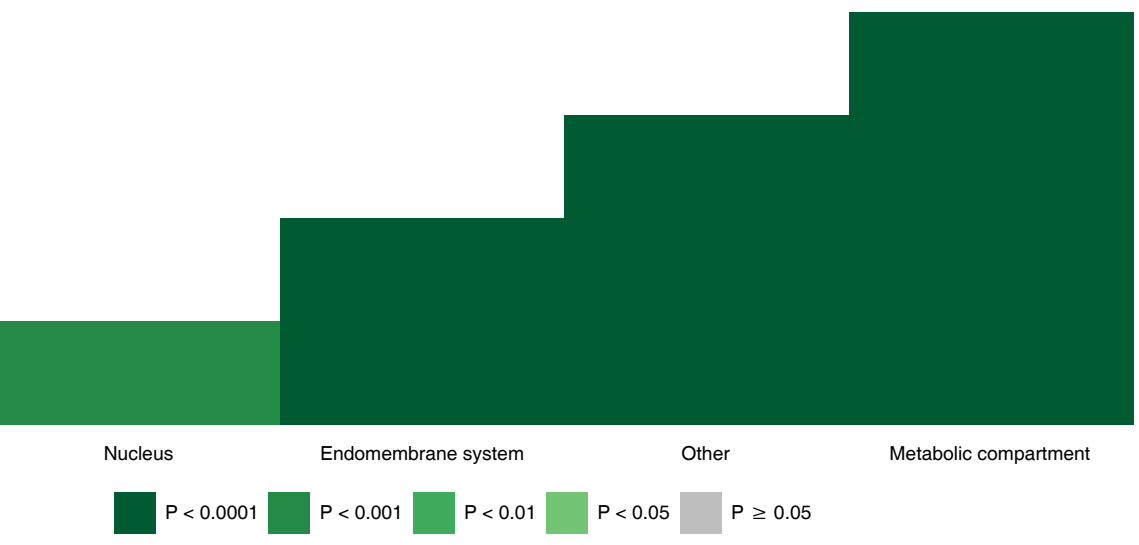

Supplementary Fig. 6 | Contribution of innovations to families with a particular cellular localisation.

Statistical significance of pairwise comparisons (Fisher's exact tests) between the proportions of LECA families being derived from a eukaryotic innovation (invention or duplication) for different localisations (a) and the corresponding broad categories (b). The values for each localisation are shown in Extended Data Fig. 3d. The axis labels are ordered based on the innovated fraction. 
a

Inorganic ions

Signal transduction

Lipids

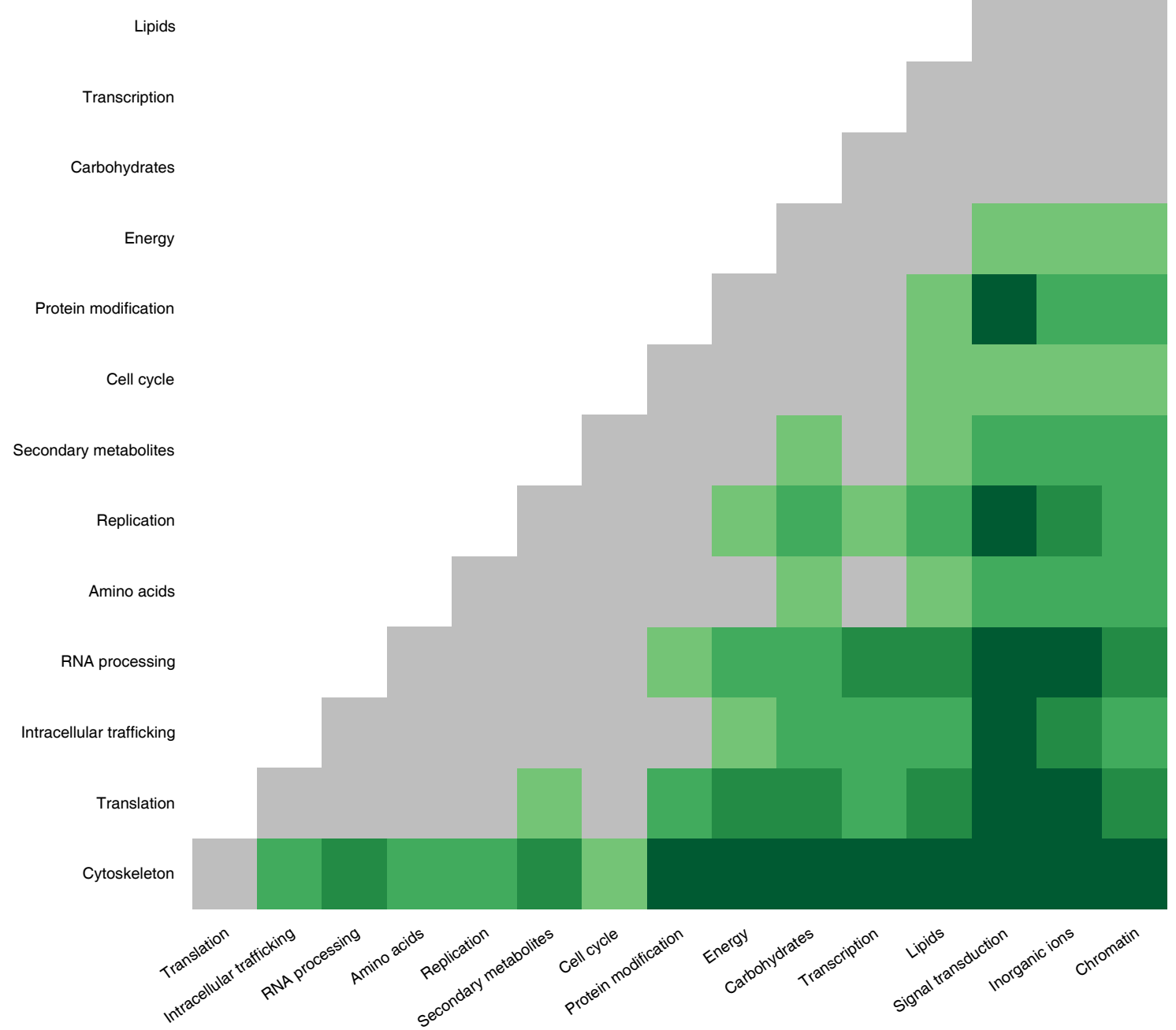

b

Cellular processes and signalling

Information storage and processing

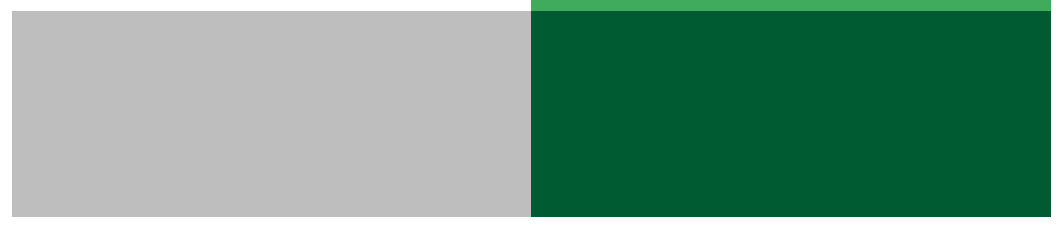

Cellular processes and signalling

Metabolism

$P<0.0001 \quad P<0.001 \quad P<0.01 \quad P<0.05 \quad P \geq 0.05$

Supplementary Fig. 7 | Comparison of duplication lengths between different functions.

Statistical significance of pairwise comparisons (Mann-Whitney $U$ tests) between the duplication lengths for different functions (see Fig. 4a) (a) and the corresponding broad categories (b). The axis labels are ordered based on the median of duplication lengths. 
a

Plasma membrane

Endoplasmic reticulum

Mitochondrion

Golgi apparatus

Endosome
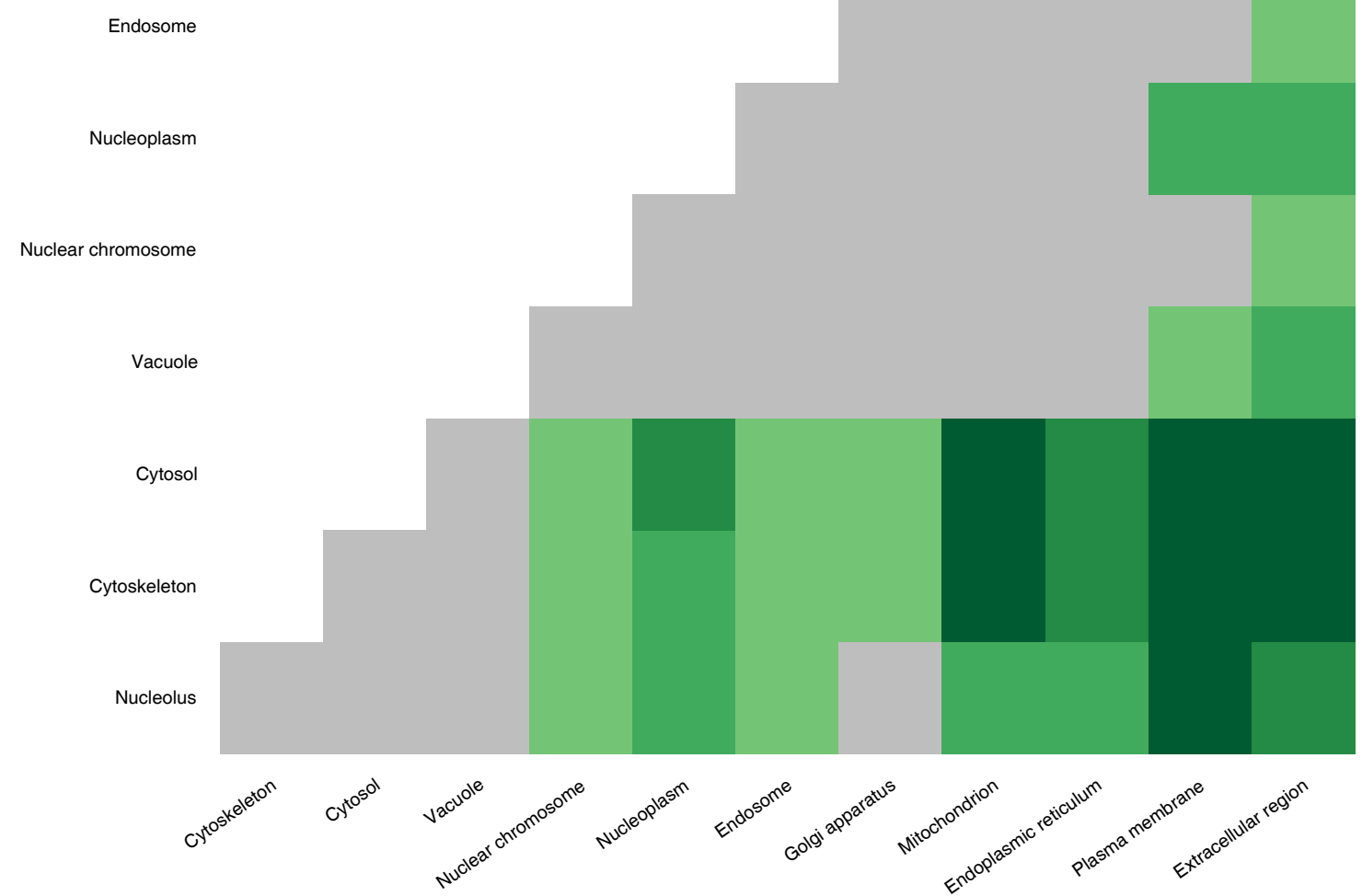

b

Metabolic compartment

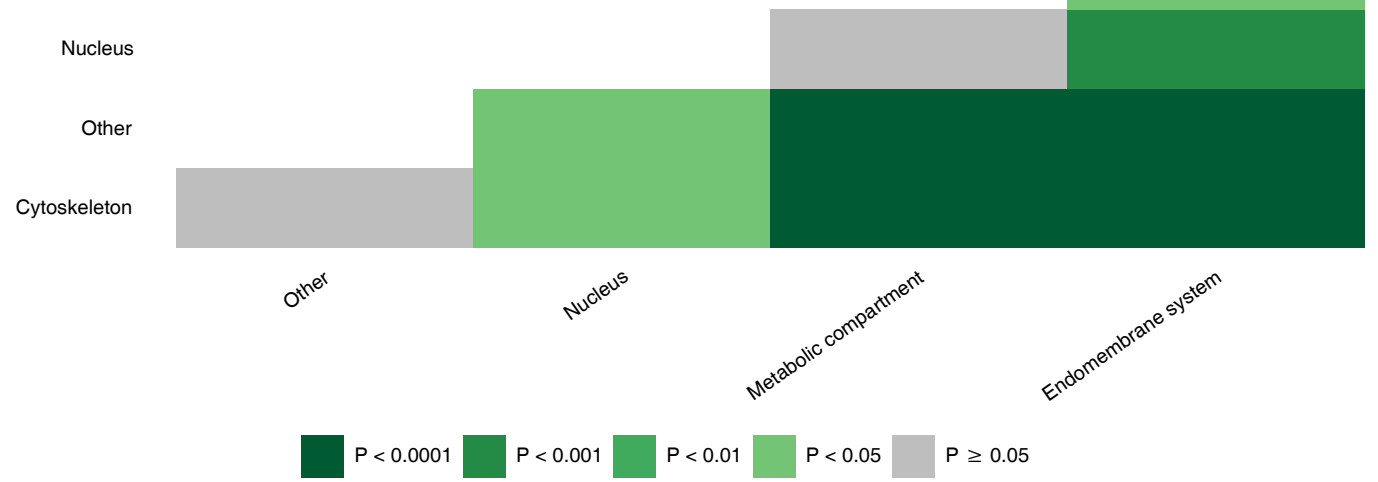

Supplementary Fig. 8 | Comparison of duplication lengths between different cellular localisations.

Statistical significance of pairwise comparisons (Mann-Whitney $U$ tests) between duplication lengths for different localisations (see Fig. 4b) (a) and the corresponding broad categories (b). The axis labels are ordered based on the median of duplication lengths. 